\title{
Implicit Personalization in Driving Assistance: State-of-the-Art and Open Issues
}

\author{
Dewei Yi*, Jinya Su, Liang Hu, Cunjia Liu, Member, IEEE, Mohammed Quddus, Mehrdad Dianati, Senior \\ Member, IEEE and Wen-Hua Chen, Fellow, IEEE
}

\begin{abstract}
In recent decades, driving assistance systems have been evolving towards personalization for adapting to different drivers. With considering personal driving preferences and characteristics, these systems become more acceptable and trustworthy. This paper presents a survey of recent advances in implicit personalized driving assistance. We classify the collection of work into three main categories: 1) personalized Safe Driving Systems (SDS), 2) personalized Driver Monitoring Systems (DMS), and 3) personalized In-vehicle Information Systems (IVIS). For each category, we provide a comprehensive review of current applications and related techniques along with the discussion of industry status, gains of personalization, application prospects, and future focal points. Several existing driving datasets are summarized and open issues of personalized driving assistance are also suggested to facilitate future research. By creating an organized categorization of the field, this survey could not only support future research and the development of new technologies for personalized driving assistance but also facilitate the use of these techniques by researchers within the driving automation community.
\end{abstract}

Index Terms-Intelligent vehicles; driver behavior analysis; personalization; Advanced Driver Assistance Systems;

\section{INTRODUCTION}

Safety, efficiency, and convenience are three key concerns raised in recent studies on intelligent vehicles [1-8]. According to a World Health Organization report, up to 50 million people are injured or disabled in road accidents worldwide every year with $90 \%$ of deaths occurred in developing nations [9]. As reported by the U.S. National Highway Traffic Safety Administration, 32,719 fatalities and 2.3 million injuries occurred in the US in 2013 [10]. In addition, according to the 2015 Urban Mobility Scorecard report, traffic congestion costs $\$ 160$ billion per year and causes the waste of three billion gallons of fuel. Moreover, the environment is polluted by vehicles' tailpipe emissions. To this end, a number of in-vehicle advanced

This work was supported by the U.K. Engineering and Physical Sciences Research Council (EPSRC) Autonomous and Intelligent Systems programme under the grant number EP/J011525/1 with BAE Systems as the leading industrial partner. (Corresponding author: Dewei $\mathrm{Yi}^{*}$ ).

Dewei Yi and Mehrdad Dianati are with Warwick Manufacting Group, University of Warwick, Coventry CV4 7AL. (e-mail: Dewei.Yi@warwick.ac.uk; M.Dianati@warwick.ac.uk).

Jinya $\mathrm{Su}$ and Liang $\mathrm{Hu}$ are with the School of Computer Science and Electronic Engineering, University of Essex, Colchester CO4 3SQ, UK. (email: j.su@essex.ac.uk; 1.hu@essex.ac.uk).

Cunjia Liu and Wen-Hua Chen are with the Department of Aeronautical and Automotive Engineering, Loughborough University, Loughborough LE11 3TU, U.K. (e-mail: c.liu5@lboro.ac.uk; w.chen@lboro.ac.uk).

Mohammed Quddus is with School of Civil and Building Engineering, Loughborough University, Loughborough LE11 3TU, U.K. (e-mail: M.A.Quddus@lboro.ac.uk). functions have been developed and implemented. In this paper, the baseline we used to classify driving assistance systems is the application domains of these systems. Typically, three application domains are considered: (i) the vehicle; (ii) the driver; (iii) the service that the vehicle provides for the driver. Corresponding to the three domains respectively, three kinds of categories are summarized for driving assistance systems as follows: (i) Safe Driving Systems (SDS), which work on the vehicle, especially on vehicle dynamics and control, are designed to reduce potential risks of accidents and even avoid collisions [11-13]. Typical functions of SDS include adaptive cruise control, collision avoidance, lane-keeping assistance, lane change assistance, and intersection assistance; (ii) Driver Monitoring Systems (DMS) are designed to supervise the status of drivers so that they can be warned about abnormal driving behaviors and mental states [14]. Typical functions of DMS include fatigue and distraction detection, driving style recognition (range prediction), and affective state recognition; (iii) In-Vehicle Information Systems (IVIS) provide in-time information and services for the driver [15]. Typical functions of IVIS include route recommendations, entertainment services recommendations, notification services, and interactive assistance.

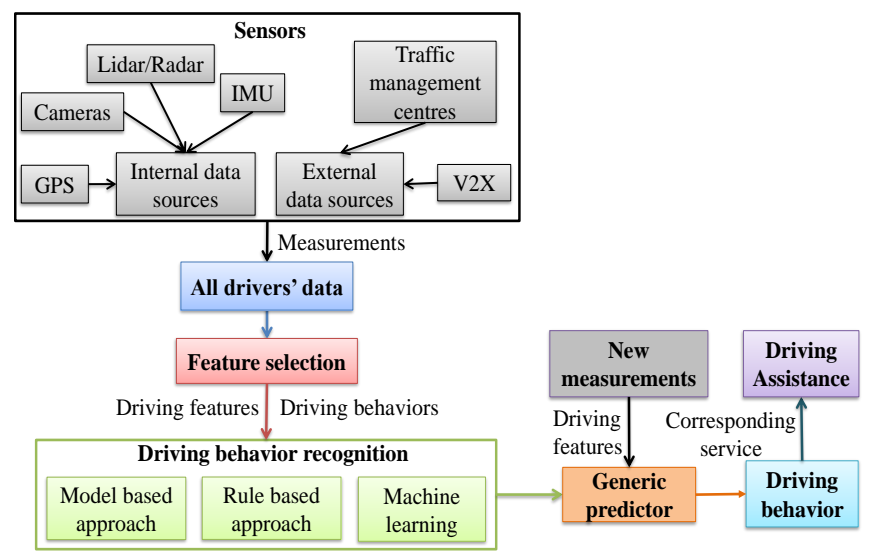

Fig. 1. Process of generic driving assistance, where V2X means vehicleto-everything (e.g. vehicle, infrastructure) communication [16 17]. Internal data sources denote data collected by vehicle embedded sensors. External data sources denote data collected by broadcasts, communicating with others vehicles and road infrastructures. "all drivers' data" imply that no driver ID is recorded in data collection.

Human factors [18] or individual driver's preferences are involved in all these systems. The common design approach for SDS, DMS, and IVIS is to develop a generic system that can work for all drivers. We show a schematic of the overall 
framework in Fig. 11. In a generic system, signals from internal data sources (the sensors embedded in a vehicle, e.g. GPS, camera, IMU, Lidar and radar) and external data sources (the data obtained from communication networks and traffic radios, e.g. traffic management centers and V2X communication) are treated indiscriminately even though these signals are from different drivers. Next, the principal features are chosen by using feature selection techniques so as to conspicuously link the driving features to the corresponding driving behaviors. After obtaining the principal driving features and labels of the corresponding driving behaviors, driving behaviors can be recognized by three different approaches including: model based approaches, rule based approaches, and machine learning approaches. The predictors of model based approaches are derived from driver models (e.g. intelligent driver model and car-following model) as in [19-21]. The predictors of rule based approaches are often used to recognize driver behaviors using a predetermined threshold [22--24]. The predictors of machine learning approaches are obtained by training a classifier or regressor (e.g. Bayesian network, decision tree, and support vector machine) as in [5, 25, 26]. Then, the predictor is used in a generic system. When the new measurements are received by sensors, the corresponding driving behaviors (e.g. fatigue, distraction) are recognized by the generic system so that corresponding services (e.g. guiding drivers to rest stops, alerting drivers) can be provided. It is noticeable that the generic approach trains or designs a model by using the driving data of all drivers indiscriminately, and, as a result, personalized driving characteristics and preferences of individual drivers may be neglected [27]. In practice, different drivers may have distinct driving characteristics and preferences even in a similar driving scenario [3]. Therefore, it is not surprising that a conventional generic approach may provide limited performance and satisfaction for individual drivers. This motivates the introduction of personalized driving assistance, implicitly embedding personalized styles, preferences, and characteristics. Here, the driving styles refer to drivers' personal feelings about whether their driving is normal, moderate or aggressive. The procedure of collecting normal and aggressive driving data for individual drivers is outlined in [28]. Driving preference and characteristic refer to personal driving behaviors such as preferred distance to the car in-front [20, 26] and adaptive lane change assistance [29].

This paper presents a comprehensive review of personalized driving assistance. Personalization of driving assistance is discussed from three different aspects, where the taxonomy and related techniques of driving assistance are presented in Fig. 2 . To the best of the authors' knowledge, this is the first attempt to conduct a comprehensive review of implicit personalized driving assistance. More precisely, the main contributions are summarized below:

- According to application domains, driving assistance systems are divided into SDS, DMS, and IVIS with the corresponding functions.

- The motivation and key components of personalized driving assistance systems are discussed.

- State-of-the-art implicit personalized driving assistance

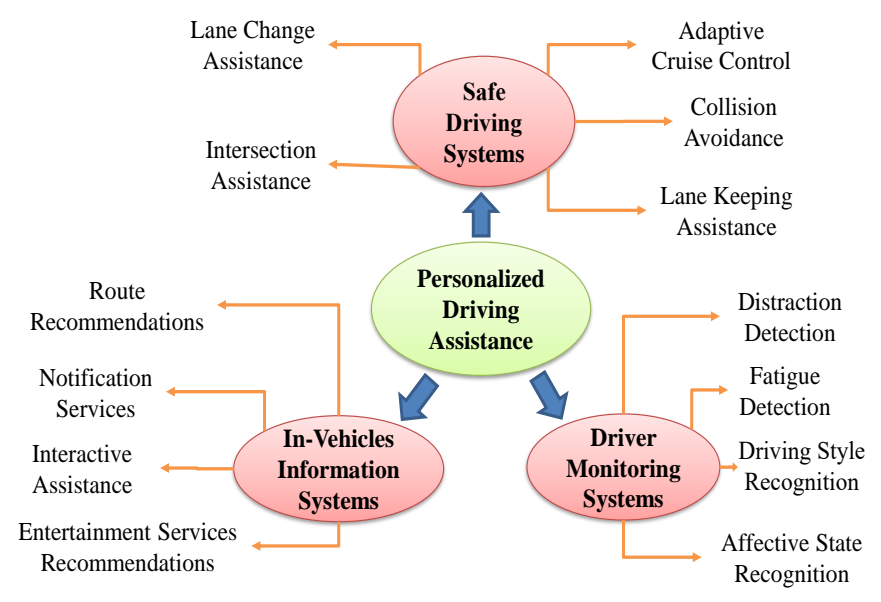

Fig. 2. The Categories of Personalized Driving Assistance.

techniques in SDS, DMS, and IVIS are elaborated along with dataset types, inputs, algorithms, pros, and cons.

- Detailed discussion is conducted on SDS, DMS, and IVIS in terms of industry status, gain of personalization, application prospects, and future focal points. The literature of SDS, DMS, and IVIS covers from 1999 to 2019, from 2009 to 2019, and from 2001 to 2019 respectively.

- Open issues on implicit personalized driving assistance are highlighted to inspire future research.

\section{Personalization in Driving Assistance}

According to [3, 22, 26, 30,-34], driving assistance systems should be safe, effective, and comfortable. To meet these criteria, personalization is introduced to understand the status of a specific driver [35], and take individual driving styles [29], requirements, and preferences [36] into account.

Personalized systems are often realized in implicit ways using data-driven approaches. This is because implicit personalization allows a system to adapt to the user through interactions and historical usage data with little direct input from the driver [37, 38]. For instance, the parameters of an intelligent driver model [39] can be tuned from individual historical driving data. The key components of the personalization process include observing the driving behaviors, modelling human driving behaviors and validating the models. The overall structure is depicted in Fig. 3. 1) Observing the driving behaviors: Individual driving behaviors can be observed from his/her historical driving data. The task in this step focuses on personal driving data collection. 2) Human driving behaviors and preferences modelling: The data of a specific driver is used to train a driver model, which is then used in either driving state recognition or vehicle dynamic control [20, 40, 41]. 3) Validation of a personalized model: Evaluation of a personalized model can be classified into four levels: a) Offline playback; b) Simulation in a traffic simulator; c) Human in the loop simulation; d) Field test [42]. Among them, the field test is most convincing. However, it is also the most challenging due to a relatively large cost and issues with safety. To this end, human in the loop simulation [32, 43] is a promising efficient and meaningful alternative. 


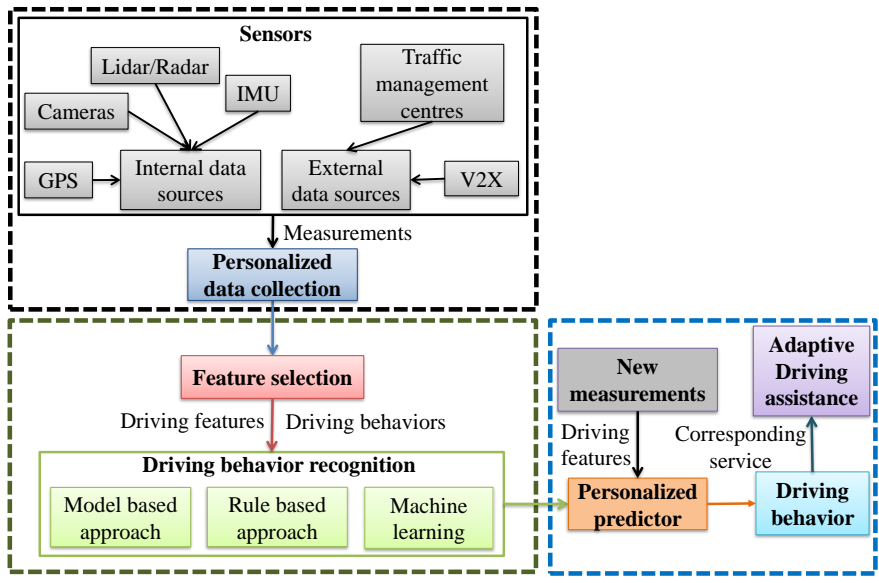

Fig. 3. Personalized process, where the blocks within black dashed lines are for observing the driving behaviors, the blocks within dark green dashed lines are for human driving behaviors and preferences modelling, and the blocks within dark blue dashed lines are for the validation of a personalized model.

\section{Personalized SAFE DRIVING Systems (SDS)}

SDS have evolved substantially in the past decades and have become a significant component of intelligent vehicles. SDS are focused on out-vehicle environment (e.g. road, other vehicles, and other road users) rather than in-vehicle environment (e.g. drivers, passengers). Therefore, "out-vehicle assistance" links more closely to vehicle dynamic control. This section reviews the related studies in five different aspects: adaptive cruise control, collision avoidance, lane keeping assistance, lane change assistance and intersection assistance. The related literature of personalized SDS, presented in this paper, is summarized in Table I] and Table II along with the description of dataset types, inputs, used algorithms, pros, and cons.

\section{A. Adaptive Cruise Control}

Adaptive cruise control focuses on the longitudinal control of a vehicle, which drives a vehicle at a pre-defined speed whilst maintaining a desired gap with the vehicle in-front. However, conventional adaptive cruise control systems only provide a limited number of pre-defined gaps. Such design makes these systems difficult to satisfy the requirements of different drivers. To overcome this weakness, a great number of personalized adaptive cruise control systems have been developed over recent decades. In [23, 47, 51, 66], personalized adaptive cruise control systems adapt to drivers in real-time based on the observation of the drivers' style and preferences. Here, artificial neural networks, linear models or a combination of the two are used to generate time gaps of a specific driver according to the driver's historical driving data. In [44], authors design a fuzzy controller based on evolutionary strategies, which can generate fuzzy rules by using the driving data of a specific driver such that a variety of behaviors can imitated with great accuracy. Different from the aforementioned approaches, learning-based approaches that use Model Predictive Control are used in [21, 53, 57, 58]. This allows them to imitate each driver's style and preferences so as to achieve personalized adaptive cruise control of a vehicle. In addition, [20] predicts a driver's throttle and braking pedal operations according to time headway and inverse time to collision. In contrast to previous research that mainly focuses on imitating a specific driver's behaviors, [18, 19, 65] reduce the errors of longitudinal control by building a personalized driving model. Driver's behaviors are modeled using a Gaussian Mixture Model approach. All in all, most of the personalized adaptive cruise control functions can provide reasonable performance. One big challenge is how to define principal features for different drivers, because different drivers have different driving characteristics and therefore useful features for different drivers may be entirely different. Inspired by [73. 74], the principal individual driving characteristics can be extracted by using model selection techniques (e.g. Wald statistics) [73] or feature selection algorithms (e.g. sequential forward floating selection) [74].

\section{B. Collision Avoidance}

Collision avoidance systems enhance driving safety by alerting drivers to an impending collision or automatic braking for avoiding potential collisions. However, different drivers have different driving styles, preferences, and characteristics. A generic model based collision avoidance approach cannot perform well for all drivers. To reduce the false alarms and extend the reaction time, personalized driving characteristics can be considered for these systems [23, 67, 68, 70, 75]. Rule based collision avoidance algorithms are intuitive approaches to predict a crash event, where a threshold for autonomous braking is learned from personalized historical driving data [23]. In [67], a statistical behavior modeling approach is proposed to estimate the danger level probability distribution of a particular driver such that an activation threshold can be determined to warn them of the potential of an emerging crash. However, the warning threshold of different driving situations should be different. Therefore, authors in [68] develop an online learning forward collision warning algorithm which adjusts the warning threshold automatically by considering the current driving situation. In contrast to the aforementioned studies, [70] implements personalized steering assistance by introducing a personalized potential field. In the proposed system, a personalized potential map is built up to represent hazard awareness of each driver. In brief, online learning algorithms can be promising solutions which can adjust the threshold of a specific driver over time. Additionally, returning uncertainty is significant for decision making on vehicle dynamics control, where systems can provide the probability of potential collision [76]. However, the approaches used here are "offline", which means they cannot tune the threshold over time as in [23].

\section{Lane-Keeping Assistance}

Lane-keeping assistance aims to alert drivers to a forthcoming lane departure. However, a failure to understand the driver's correct behavior may cause a significant number of false warnings. This could make drivers mistrust or even abandon lane-keeping assistance systems [26, 92]. To reduce false positive rate, Hidden Markov Models, Gaussian Mixture 
TABLE I

Summary of the Presented Research in Personalized SDS (Part A)

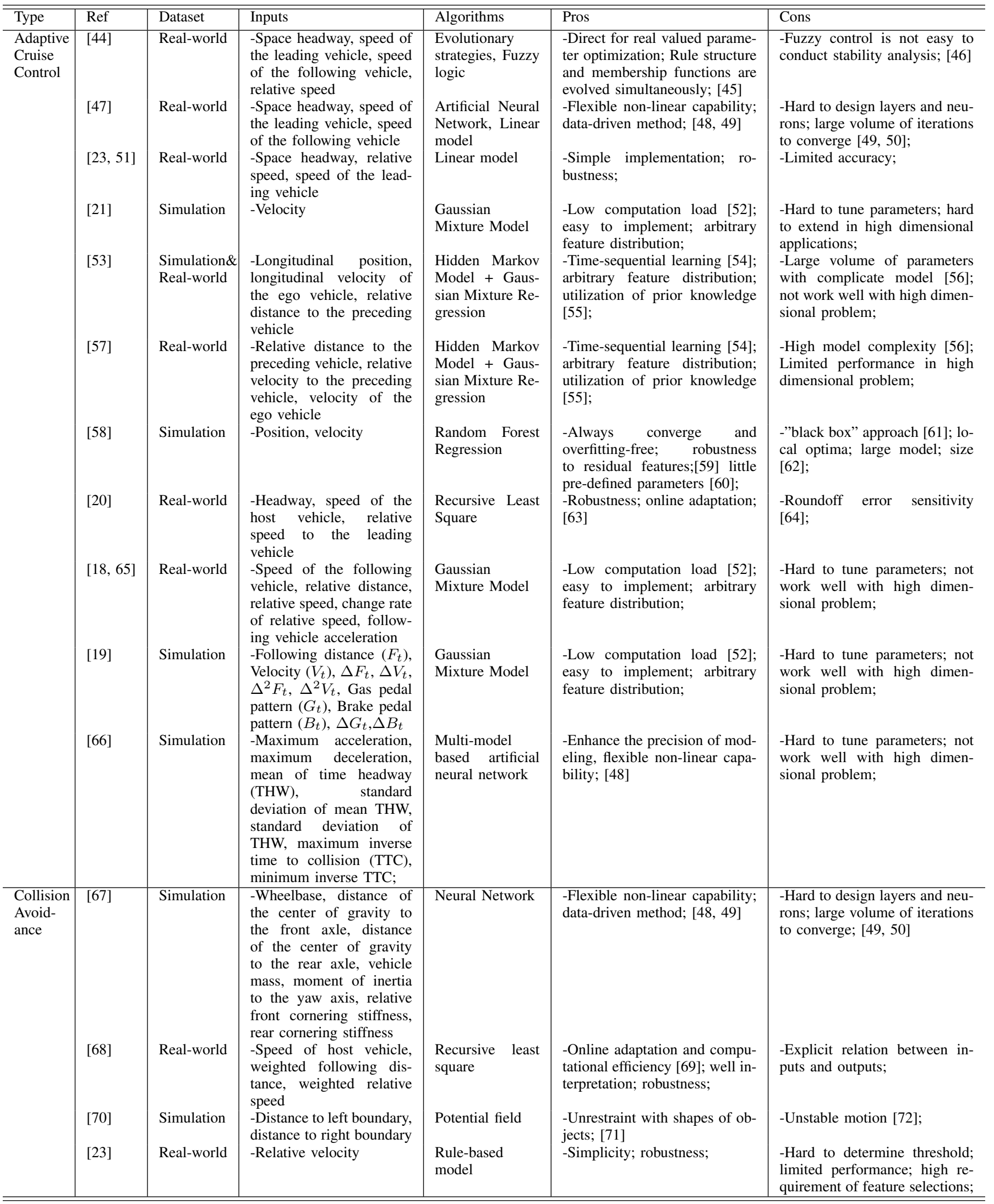


TABLE II

Summary of the Presented Research in Personalized SDS (Part B)

\begin{tabular}{|c|c|c|c|c|c|c|}
\hline Type & Ref & Dataset & Inputs & Algorithms & Pros & Cons \\
\hline $\begin{array}{l}\text { Lane- } \\
\text { Keeping } \\
\text { Assistance }\end{array}$ & [57] & Real-world & $\begin{array}{l}\text {-Longitudinal velocity, } \\
\text { distance to the lane } \\
\text { center (y), orientation } \\
\text { with respect to the lane } \\
\text { center }(\theta) \text {, derivative of } \\
y \text {, derivative of } \theta \text {, road } \\
\text { curvature } \\
\text {-Vehicle speed, relative } \\
\text { yaw angle, relative yaw } \\
\text { rate, road curvature, lat- } \\
\text { eral displacement }\end{array}$ & $\begin{array}{l}\text { Hidden Markov } \\
\text { Models + Gaus- } \\
\text { sian Mixture Re- } \\
\text { gression }\end{array}$ & $\begin{array}{l}\text {-Time-sequential learning [54]; } \\
\text { arbitrary feature distribution; } \\
\text { utilization of prior knowledge } \\
\text { [55]; }\end{array}$ & $\begin{array}{l}\text {-Large volume of parameters } \\
\text { with complicate model [56]; } \\
\text { not work well with high dimen- } \\
\text { sional problem; }\end{array}$ \\
\hline \multirow[t]{6}{*}{$\begin{array}{l}\text { Lane } \\
\text { Change } \\
\text { Assistance }\end{array}$} & 27] & Simulation & $\begin{array}{l}\text {-Distance of gap, relative } \\
\text { speed of interest } \\
\text {-Steering wheel angle, the } \\
\text { error between desired path } \\
\text { and current path }\end{array}$ & $\begin{array}{l}\text { Gaussian } \\
\text { Mixture Models } \\
\text { Lateral driver } \\
\text { model }\end{array}$ & $\begin{array}{l}\text {-Low computation load [52]; } \\
\text { easy to implement; arbitrary } \\
\text { feature distribution; } \\
\text {-Intuitive interpretation; easy } \\
\text { realization; }\end{array}$ & $\begin{array}{l}\text {-Hard to tune parameters; lim- } \\
\text { ited performance in high di- } \\
\text { mensional problem; } \\
\text {-Hard to guarantee accuracy; }\end{array}$ \\
\hline & [78 & Simulation & $\begin{array}{l}\text {-Distance of ego-car (E) } \\
\text { and merging-car }(\mathrm{M}) \text {; rel- } \\
\text { ative velocity between E } \\
\text { and M; relative accelera- } \\
\text { tion between E and lead- } \\
\text { ing car; relative distance } \\
\text { to the end of acceleration } \\
\text { lane; length of recogniz- } \\
\text { able area; }\end{array}$ & $\begin{array}{l}\text { Decision entropy } \\
\text { +Randomized } \\
\text { Model Predictive } \\
\text { Control+logistic } \\
\text { regression model }\end{array}$ & $\begin{array}{l}\text {-Low computation load }[52] \\
\text { easy to implement; take human } \\
\text { drivers' preferences and uncer- } \\
\text { tainty into account; }\end{array}$ & $\begin{array}{l}\text {-Neglect the personality and } \\
\text { preferences of drivers; }\end{array}$ \\
\hline & 80] & Simulation & $\begin{array}{l}\text {-Longitudinal Vehicle } \\
\text { Speed, yaw angle, lateral } \\
\text { Deviations, steering wheel } \\
\text { angle }\end{array}$ & $\begin{array}{l}\text { Logistic regres- } \\
\text { sion model }\end{array}$ & $\begin{array}{l}\text {-Easy to implement; Fast run- } \\
\text { time [52]; } \\
\text {-Feedback-free [81; }\end{array}$ & $\begin{array}{l}\text {-The diversity of the partici- } \\
\text { pants is not enough (it had bet- } \\
\text { ter include drivers from differ- } \\
\text { ent age groups and genders); } \\
\text {-Slow response; unstable; } 81\end{array}$ \\
\hline & 82] & Simulation & -Electroencephalography & $\begin{array}{l}\text { Extend queuing } \\
\text { network }\end{array}$ & -High stability [83]; & $\begin{array}{l}\text {-Low robustness (single source) } \\
\text { [84]; }\end{array}$ \\
\hline & 85 & & $\begin{array}{l}\text {-Speed, proximities to in- } \\
\text { ner/outer road boundary }\end{array}$ & $\begin{array}{l}\text { Inverse optimal } \\
\text { control }\end{array}$ & -Constructive; stability; [86] & $\begin{array}{l}\text {-Model-dependent; } \\
\text { dependent; }[87]\end{array}$ \\
\hline & 88 & Real-world & $\begin{array}{l}\text {-Velocity, relative velocity } \\
\text { and distance }\end{array}$ & $\begin{array}{l}\text { fuzzy c-mean } \\
\text { clustering }+ \\
\text { fuzzy knn }+ \\
\text { intelligent driver } \\
\text { model }\end{array}$ & $\begin{array}{l}\text {-Labeling-free and model-free; } \\
\text { easy to implement; arbitrary } \\
\text { feature distribution; }\end{array}$ & $\begin{array}{l}\text {-Hard to choose distance crite- } \\
\text { ria in feature space and tune } \\
\text { the threshold for convergence; } \\
\text { High computation load; }\end{array}$ \\
\hline \multirow[t]{3}{*}{$\begin{array}{l}\text { Intersection } \\
\text { Assistance }\end{array}$} & 89] & Simulation & $\begin{array}{l}\text {-Traffic lights location and } \\
\text { timing data for each one } \\
\text { of them on the route, } \\
\text { traffic flow speed (V2I } \\
\text { needed), fuel consump- } \\
\text { tion, time of arrival }\end{array}$ & $\begin{array}{l}\text { Sequential } \\
\text { Quadratic } \\
\text { Programming }\end{array}$ & $\begin{array}{l}\text {-Flexibility; non-linear models; } \\
\text { multiple objectives; } 90\end{array}$ & -High computation load [90]; \\
\hline & 91 & & & $\begin{array}{l}\text { Maximum Like- } \\
\text { lihood }\end{array}$ & $\begin{array}{l}\text {-Consistent parameter estima- } \\
\text { tion; solid theoretical basis; }\end{array}$ & $\begin{array}{l}\text {-Biased for small samples; lo- } \\
\text { cal optima; }\end{array}$ \\
\hline & 23] & Real-world & -Relative velocity & $\begin{array}{l}\text { Rule based } \\
\text { model }\end{array}$ & -Simplicity; robustness; & $\begin{array}{l}\text {-Thresholds and features selec- } \\
\text { tion; limited performance; }\end{array}$ \\
\hline
\end{tabular}

Models, and their combination are used in personalized lanekeeping assistance systems [57, 77]. These systems can learn a driver's preferences when a human-driver keeps driving in a lane. Subsequently, these systems accommodate to each driver by considering his/her driving preferences and characteristics. In general, the Gaussian Mixture Models is robust to the feature distribution and is able to deal with non-linear problems. Hidden Markov Models can process sequential data (or streaming data). It is not surprising that their combination, which inherits the advantages of Gaussian Mixture Models and Hidden Markov Models, outperforms both of them.

\section{Lane Change Assistance}

Lane changing is one of the most challenging tasks during driving. This is because it not only requires drivers to have a clear perception and projection of the surrounding environment, but also involves changes in the longitudinal and lateral speed of the vehicle. To make lane change assistance more acceptable and effective, the driving characteristics of a specific driver need to be accommodated, as suggested by [27, 29, 78. 80, 82, 85, 88, 93]. In [29], Gaussian Mixture Models are used to adjust the kinematic model parameters so as to adapt to individual driving styles. Moreover, authors in [88] achieve better gap prediction with considering the characteristics of 
drivers. Here, the fuzzy c-mean clustering algorithm is combined with Kalman filter to estimate the distance from following vehicle to the heading vehicle more accurately. Another approach implements personalized lane changing by proposing a compensatory transfer function based on a driver model in combination with a feedforward anticipatory subsystem [27]. Furthermore, [85] learns a driver's steering characteristics by using inverse optimal control. In this research, inverse optimal control is used to identify the parameters of a cost function, where the cost function is designed by considering speed, steering, and the inner/outter road boundary. In addition, lane change assistance plays a significant role in merging tasks. In [78, 79], logistic regression models are used to determine the acceptability of merging tasks. Compared to [79], [78] also takes preferences of drivers on the main lane into account, which is achieved by minimizing decision entropy. Such design makes driving assistance more acceptable and efficient. Lane change assistance is a sharing control task, where a human driver and the vehicle controller are able to collaborate with each other. To this end, [80, 93] develop a Human-Centered Feed-forward Control system, where a driver's steering characteristics and the human driver's steering inputs are both taken into account for vehicle steering control. More exciting research in personalized lane change assistance is to predict steering angle by the electroencephalography signal[82]. This study shows that a human driver's intention can be reflected by his/her electroencephalography signal.

\section{E. Intersection Assistance}

Intersection crossing is one of the most frequent driving maneuvers in urban and metropolitan areas. To make intersection assistance more desired, several intersection assistant systems are proposed with the consideration of personal driving preferences [23, 89, 91]. The distance of braking or the distance required to release the accelerator can be expressed by a polynomial regression model, where the coefficients of the model are calibrated by personal driving data in order to adapt to different drivers [23]. In [89], the authors propose a personalized pace optimization algorithm to help drivers approach and cross through a signalized interaction. The proposed algorithm optimizes pace on a route by considering driver characteristics so that fuel use and waiting time are minimized. Different from conventional methods (e.g., Troutbeck [94], Raff [95]), authors in [91] estimate a critical gap by using Maximum Likelihood Estimation. The critical gap is the smallest acceptable gap for a specific driver. According to experimental results, the false alarm rate can be reduced from $11.8 \%$ to $9.8 \%$ by introducing the critical gap. Overall, the polynomial regression model is a feasible approach to predict braking and accelerator release behaviors. However, are there any better models to describe these behaviors? For instance, the Gaussian Process may provide a better model for these behaviors, which has the additional advantages of providing confidence intervals and not requiring the order of the regression model to be defined a priori [96]. Furthermore, Maximum Likelihood Estimation is numerically stable and straightforward to implement.

\section{F. Discussion}

Industry status: Adaptive cruise control functions are provided by many models of cars (e.g. Audi A8, Volkswagen Touareg, BMW 5 and 6 series) [97]. Similarly, collision avoidance systems have also been successfully used in many brands and models such as Audi (A8, A7, A3), Dodge Durango, Honda (Accord, Inspire), Lexus (LS, GS, IS, RX), Skoda Octavia, Tesla Model S [97]. However, these functions are often implemented using rule-based approaches, which cannot adapt to individual drivers in an online manner. Although lots of studies have been conducted on personalized SDS, automotive manufacturers have not rushed to promote personalized functions of SDS. This may be because integrating the personalized learning algorithms into existing SDS needs careful testing to guarantee compatibility and security.

Gains of personalization: Safe driving systems can obtain several benefits by introducing personalization. The primary gain is the enhanced acceptability [26, 68]. In [26], the false-warning rate of a lane departure warning system can be reduced to $3.13 \%$. In [68], the false positive rate of a forward collision warning system is decreased below $10 \%$. The secondary gain is safety. When the false alarm is too high, the systems can become annoying to drivers and may be abandoned [18, 29]. Therefore, the enhanced acceptability can encourage drivers to keep SDS, which leads to an improvement in driving safety.

Application prospects: In adaptive cruise control, recursive least square and Gaussian mixture models are the two most promising approaches and have been used in real-time vehicle tests [18, 20, 65]. Other approaches in [44, 47, 53] have potential, but so far have only been validated using offline playback. In collision avoidance, recursive least squares is feasible to be commercialized by automotive companies. Different from [23], recursive least squares does not only overcome the online adaptation issue but also can be run in real-time on a test vehicle [68]. In lane-keeping assistance, not many studies have used real-time vehicle testing. According to the real-world data playback validation results, the combination of hidden Markov models and Gaussian mixture models (or regressions) [26, 57] are promising approaches. In lane change assistance, Gaussian mixture models [29] are a suitable approach. Compared to the data-driven intelligent driver model mentioned in [88], Gaussian mixture models do not need a large volume of data at the beginning and can adapt to individual drivers online. In intersection assistance, for now, maximum likelihood estimation and linear approximation are the two feasible approaches [23, 91]. Compared to the maximum likelihood method which is only validated in simulations [91], linear approximation is more practical since it can be validated by real-world data playback [23]. When the vehicular communication devices and road communication facilities are more sound and ubiquitous, sequential quadratic programming may become practical and effective. For the time being, however, the performance of sequential quadratic programming is only assessed in a simulation environment.

Future focal points: Firstly, safe interaction amongst users (human drivers or even autonomous vehicles) on the road 
needs to be prioritized [98]. The implementation of safe interaction is challenging because human actions and behaviors are often unpredictable [99]. Fortunately, studies in [98, 100] provide some promising ideas (such as developing robust informative models or regenerative stochastic models). Secondly, intersection assistance may become a focal point with the development of vehicle embedded devices (e.g. communication modules, high-performance CPU/GPUs) and road infrastructures (e.g. roadside units), which can not only make approaching an intersection safer and more smooth (for example, by reducing unnecessary braking and providing collision warnings), but also provide clearer communication amongst drivers to improve the fluency of their interactions.

\section{Personalized Driver Monitoring Systems} (DMS)

In recent years, in-vehicle monitoring systems have been developed rapidly and pervasively applied in healthcare and cognitive workload recognition [101]. Driver monitoring systems can detect abnormal driving behaviors (drowsiness, fatigue, distraction) or driving styles (normal, moderate, aggressive) via vehicle dynamic measurements or vision measurements. Moreover, driver monitoring systems are one of the most significant components of vehicular safety applications detecting fatigue, distractions and the driving style/mental state of a driver [102]. However, several challenges, such as trust, acceptance, and unpredictability [98, 103, 104], may slow down the development of these systems. To overcome these issues, personalized driver monitoring may be a promising solution, which makes driving assistance more trustworthy and acceptable. Moreover, driving performances of different drivers are quite different even in the same driving scenarios. The limited feedback of personalized driving behaviors make it difficult to evaluate the performance of plug-in hybrid electric vehicles [105]. Personalized driver monitoring systems are to detect abnormal behaviors and driving styles based on individual drivers. For instance, the heart rate and blood pressure are two popular measurements to assess abnormal driving behaviors (drowsiness, fatigue, distraction) [24, 106, 107]. However, classifying based on average statistics of these two measurements easily leads to a higher false positive rate, especially for drivers with cardiovascular diseases. Because of this, personalized driver monitoring systems urgently need to be developed. Compared to SDS, the personalization in driver monitoring systems has not attracted significant attention in the past decade. Table III summarizes the relevant techniques in personalized driver monitoring systems along with the description of dataset types, inputs, used algorithms, pros, and cons.

\section{A. Fatigue and Distraction Detection}

Driver inattention monitoring can be classified into distraction and fatigue [123]. Some studies attempt to detect fatigue and distraction via video [40, 41, 109]. Vision measurements contain eye blink duration, nodding frequency, and head poses. These measurements have been proved useful to detect abnormal driving behaviors [123]. However, vision measurements are often obtained using computer vision techniques which are sensitive to light condition. Moreover, the privacy issue involved in vision also needs to be addressed. Compared to vision measurements, vehicle dynamic measurements are more robust against light condition [3]. Vehicle dynamic measurements include steering angle, lateral acceleration, longitudinal acceleration, vehicle velocity amongst others. Moreover, more features can be generated by using vehicle dynamic measurements such as steering entropy, steering reversal rate, and speed prediction error. In [108], speed prediction error and steering entropy are used as features to train a support vector machine, which can achieve high overall accuracy of $95 \%$ and a false positive rate about $78.3 \%$ based on a specific driver's data. It is found that a personalized drowsiness detection system outperforms the average system when sufficient personalized data is available for training the classifier. Personalized data collection is always challenging in a personalized application. In [101], a personalized monitoring system is proposed, where captive electrocardiogram and ballistocardiogram data can be obtained in real-time and recognize fatigue. In contrast to [101], eye blink activities are also considered in [24] and therefore the false alarms of fatigue detection can be reduced.

\section{B. Driving Style Recognition}

Range prediction and fuel management are closely related to driving styles. Moreover, driving style recognition also plays a significant role in driving safety and vehicle security. Due to the diversity of driving preferences among different drivers, the accurate evaluation of fuel consumption is a challenging task for intelligent vehicles, especially with plug-in hybrid electric vehicles [22]. To predict fuel use more precisely, various personalized vehicle energy consumption prediction approaches are proposed [32, 43, 105, 112, 114, 118]. Authors in [105] develop a personalized multi-modality sensing and analysis system, which can efficiently extract information of user-specific driving behaviors and a hybrid electric vehicle operation profile. User-specific driving behavior messages (e.g., speed, acceleration, road and traffic conditions) are fused by wavelet-based disorientation compensation to obtain accurate vehicle movement information. Hybrid electric vehicle operation profile messages (e.g. fuel use, battery system information) are used to identify the driver operation mode via classification and regression tree. The proposed approach can predict fuel use accurately (0.88- 0.996 correlation and $87.8 \%$ $89.9 \%$ classification accuracy) which is evaluated with realworld experiments. In [112, 118], the personalized DistanceTo-Empty prediction is achieved by using participatory sensing data. Various approaches are implemented and compared including a speed profile similarity matching approach, a driving habit similarity matching approach and a collaborative filtering approach. According to the experimental results, the driving habit similarity matching approach outperforms the others. Unnecessary braking and sharp acceleration cause unwanted fuel consumption, especially in approaching a traffic signal. To avoid this unnecessary fuel consumption, a scenario tree based stochastic model is introduced to adapt to a specific driver so that vehicle acceleration and braking can be reduced [114]. 
TABLE III

Summary of the Presented Research in Personalized Driver Monitoring Systems

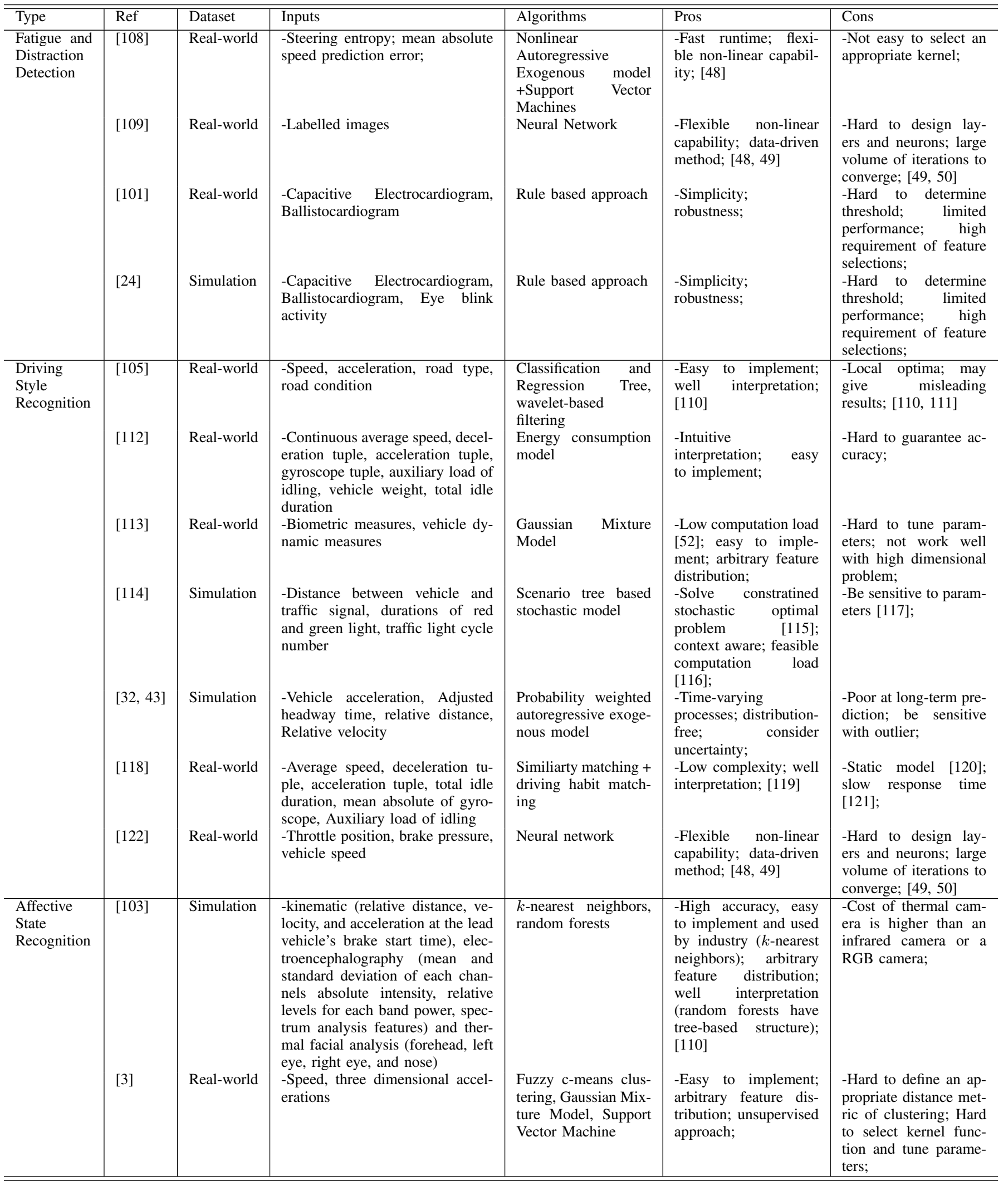


In [32, 43], probability weighted autoregressive exogenous models are used to learn individual driving behaviors for a specific driver so that fuel consumption can be estimated more precisely. Driving style and state are also important in driving safety and vehicle security. In [122], a neural network is trained to build a customized driver model for recognizing abnormal driving such as drunk driving detection. In [113], Gaussian Mixture Models are utilized to extract features which can effectively infer the driver's identification via vehiclerelated measures.

\section{Affective State Recognition}

Affective state recognition is another significant direction for human-in-the-loop systems, especially in personalized ADAS. In [103], features related to predicting the brake reaction time of the driver are generated by analyzing kinematic, electroencephalography, and thermal facial data. Taking affective sensing into account, the precision can be enhanced from $10 \%$ to $40-50 \%$. Moreover, in order to adapt to different drivers, the fuzzy c-means clustering algorithm is adopted in [3] to achieve personalization and then Gaussian mixture models and support vector machines are compared to find out the best combination to recognize driver workload.

\section{Discussion}

Industry status: In recent years, automobile manufacturers have tended to pay more attention to DMS. Honda proposes a project called Honda's automated assistant (HANA) to adjust control performance based on driver state, where driver state is measured by features such as facial expressions, voice, and heart rate [103]. Likewise, the "Sixth Sense" project of Jaguar Land Rover also intends to detect driver's stress and alertness by measuring the driver's heart rate, respiration rate, and brain activity [103]. In addition, other automobile manufacturers also develop their own DMS, including Audi (Rest Recommendation System), BMW (Active Driving Assistant), Bosch (Driver Drowsiness Detection), Ford (Driver Alert), Volkswagen (Fatigue Detection System), and Volvo (e.g. Driver Alert Control) [97]. However, all of them attempt to build an average system rather than a personalized system.

Gains of personalization: DMS can obtain several benefits by introducing personalization. The primary gain is the improved safety [108]. In [108], the driver's state (i.e. distracted or attentive) can reach a high overall accuracy of $95 \%$ when the classifier is trained on individual driver data. A secondary gain is efficiency, especially in the distance-to-empty prediction. By introducing personalization, the prediction error of distance-to-empty can be reduced to 5\% [118].

Application prospects: In fatigue and distraction detection, the combination of nonlinear autoregressive exogenous models and support vector machines is a practical approach. The required features of such approaches are easy to access and its performance is validated by a test vehicle in real-time [108]. It may be insufficient to detect drowsiness purely by eye blinking. For instance, Carsafe can only achieve $60 \%$ detection rate for drowsy driving events. To achieve a high sensitivity in monitoring driver state, the measurements of electrocardiography and electroencephalography are combined with eye blinking detection. However, it is only proved by using a driving simulator and the cost of electroencephalography sensors are also a concern for automobile manufacturers. In driving style recognition, compared to biometrics-based signals [113], participatory sensing signals (e.g. mobile measurements, geographic penetrations) are easy to access using existing navigation systems (e.g. Google Maps and Waze). In [118], a similarity matching approach based on driving habits from participatory sensing data proves to be a practical solution of range prediction for electric vehicles, which is validated by off-line playback. In state recognition (e.g. workload levels, emotions), random forests [103], $k$-nearest neighbors [103], and support vector machines [3] are promising methods. Among them, random forests and support vector machines may be more practical because the computation load of $k$ nearest neighbour increases rapidly with the increase of data dimensions and size. The recognition accuracy of random forests can achieve $86.7 \%$ by considering vehicle kinematics, thermal facial analysis, and electroencephalography together.

Future focal points: Firstly, affective state recognition should be a research emphasis due to its significance for developing provably safe human-in-the-loop systems, especially for ADAS [104]. Secondly, online unsupervised learning systems should be developed for personalized DMS. There are two main reasons: (1) manually labeling a large volume of personal data is painful and inefficient so unsupervised methods are required to achieve auto-tagging; (2) the personal driving characteristics may change with accumulation of more driving experience which needs to adapt to individual drivers in an online way.

\section{Personalized In-Vehicle Information Systems} (IVIS)

IVIS not only can provide navigation services, but also offer valuable information to drivers (e.g. traffic conditions, time delays, and alternative routes), entertainments services (e.g. music recommendation). Moreover, it can determine when, how and which services should be provided based on the current situation, which makes services more acceptable and efficient. In contrast to SDS and DMS, IVIS concentrate on in-vehicle services including route and entertainment services recommendations, notification services and interactive assistance. Table IV summarizes categories of the relevant research literature in personalized IVIS with dataset types, inputs, used algorithms, pros, and cons.

\section{A. Route Recommendations}

Route recommendations are the most common applications in IVIS. However, previous studies only care about traveling time and hardly consider business hours and the visit duration of each Point Of Interest in the route selection process, such as its attractiveness, operation hours, and order of visit [33]. Therefore, personalized interactive and traffic-aware trip planning services have attracted interest in both the academic community and in industry. TRIPPLANNER achieves personalized, interactive and traffic-aware trip planning by combining 
TABLE IV

Summary of the Presented Research in Personalized IVIS

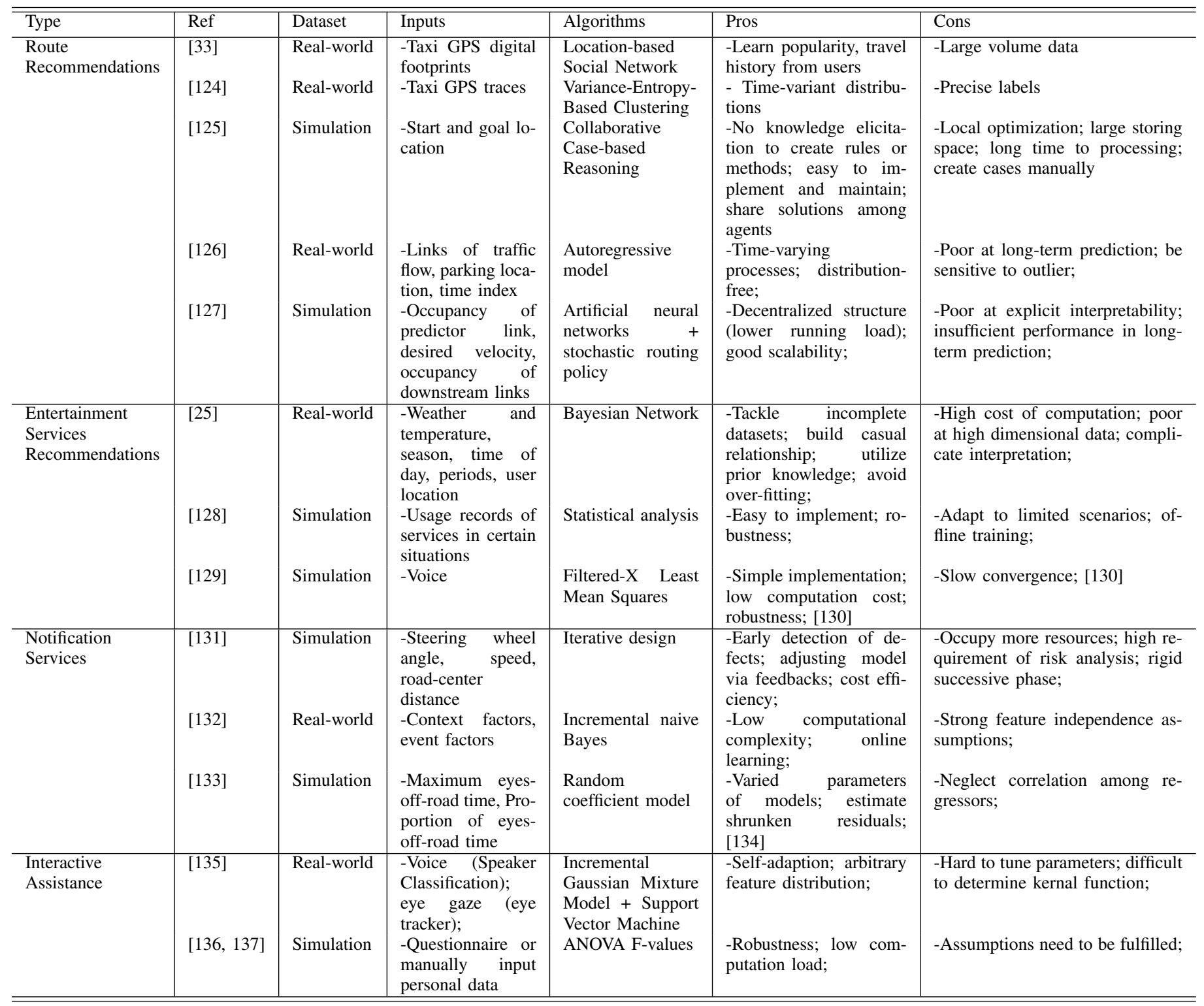

location-based social network and taxi GPS digital footprints [33]. In [124], driving behaviors of taxi drivers and endusers are learned by Variance-Entropy-Based Clustering to adapt to individual requirements, such that personalized route recommendations service can be provided to customers. Additionally, it is extremely challenging to provide personalized routes in unfamiliar territory. To mitigate this problem, [125] shares problem-solving experiences amongst multiple agents using a collaborative case-based reasoning framework to help adapt parking guiding to an individual driver's personal preferences. In [126], personalized routing instructions of parking guidance are generated by using an autoregressive model which is able to reduce, amongst other things, driving stress, as well as saving fuel. With the development of the vehicle network, road users can share their in-vehicle information such as intended destination (e.g. location) and vehicle state (e.g. speed). To this end, [127] meets individual requirements by using other vehicles' information, where an artificial neural network is combined with stochastic routing policy to generate personalized routing recommendations.

\section{B. Entertainment Services Recommendations}

It is significantly important to provide a driver with a proper service at the right location and time, however a driver's preferences should also be taken into account, especially in mobile applications [25]. In [131], a multi-modal proactive recommendation system is proposed that provides drivers with personalized content, termed "Volvo Intelligent News". "Volvo Intelligent News" system presents driver information based on the driver state and driving situation. The driver state and driving situation are obtained using driver sensors, vehicle sensors, and environmental sensors. The authors of [128] develop an intelligent In-Car-Information Systems, which is able to automatically execute an in-car-information function 
according to driver preferences in certain situations. It is achieved by integrating a contextual personalized shortcut method and a contextual personalized automation method. To provide media choice for a specific user, a Personalized Audio Zone system is designed that prevents cacophony by using Filter-X Least Mean Squares [129].

\section{Notification Services}

Notification services (e.g., calendar reminders, message and email alerts, callback reminders and news feeds) for the invehicle environment should be user-adaptive and contextaware to different drivers so as to guarantee safety and efficiency. In [132], an intelligent notification system is developed to provide an Intelligent Callback Reminder service, where incremental naive Bayes is utilized to understand the driver's situation for providing callback reminder at a right time. It is found that text entry tasks tend to increase glance duration whereas text reading tasks do not, and random coefficient models can reliably estimate individual performance when significant differences exist among different drivers [133]. These two findings are able to guide the design of personalized in-vehicle technologies.

\section{Interactive Assistance}

To cooperate with driver seamlessly and naturally, digital driving assistants should be able to recognize emotions or states of a specific driver by using speech and video as indicated by [135-137]. In [136, 137], an in-car assistant robot is developed to interact with a driver socially. Therefore, the robot can understand a driver's requirements better so as to provide proper assistance. It does not only improve the individual driving experience but is able to explore deep personalization for a specific driver over time.

\section{E. Discussion}

Industry status: IVIS do not just provide radio or entertainment or navigation, but also combinations of all of these. VOLOV develops a proactive recommendation system called "Volov Intelligent News" to present information at the appropriate time [131]. In addition, other automotive companies have developed lots of speech recognizers (such as BMW Voice Control System, Nissan Pivo, Audi AIDA, Ford Model $\mathrm{U}$ ) to enhance interaction between driver and IVIS [138]. In addition, internet companies (e.g. Google, Apple) develop IVIS related APPs (Apple CarPlay, Android Auto) to enhance human-machine interaction [138]. However, the performance of recommender systems (e,g. entertainment services, notification services) requires further improvement. Online learning mechanisms need to be integrated into IVIS so that a driver's requirement can be adapted continuously.

Gains of personalization: IVIS can obtain several benefits by introducing personalization. The primary gain is the improved efficiency [124]. In [124], on average, 50\% of routes can be achieved at least $20 \%$ faster than the competing approaches by taking personalization into account. The secondary gain is the enjoyment, where entertainment services (e.g. music, radios) and recommendation services (e.g. restaurants, scenic spots) can be provided at the right time and in the appropriate place [25, 129]. More precisely, personalized recommender system can achieve a $19 \%$ deviation from baseline driving, which outperforms the generic systems.

Application prospects: In route recommendations, TRIPPLANNER [33] is a promising solution and its efficiency and effectiveness is quantitatively evaluated in terms of computation time cost and route score using a large real-world dataset (more than 391900 passenger delivery trips in six months). In entertainment service recommendations, Bayesian networks [25] and filtered-X least mean squares [129] are two practical solutions, which are fast, well-understood, easy to implement, and tested on a real-world dataset. For entertainment service recommendations, playback is a common and effective method to evaluate performance [128]. In notification services, iterative design is applied in the "Volvo Intelligent News" system, but the system is only tested by a simulator [131]. Compared to [131], the incremental naive Bayes approach is better. This learns a driver's preferences incrementally and is embedded into an Android App, named smartNoti. In interactive assistance, compared to explicit personalization [136, 137] which relies on manual setting, implicit methods (e.g. the combination of incremental Gaussian mixture models and support vector machines [135]) are more convenient and efficient which is demonstrated in real-time vehicle tests.

Future focal points: Firstly, social interactive assistance may attract more attentions. Nowadays, the interaction between driver and IVIS is achieved by speech recognition and eye tracking [135], which is only partially capable of understanding the driver's intentions and behaviors. Social interaction needs IVIS to have a cognitive understanding of drivers. For example, the moods (e.g. anger, frustration, and sadness) of drivers should be further explored to provide the appropriate interaction (such as pacifying drivers). Second, personalized on-demand notification and recommendation services are more advanced, which can not only provide services based on personal preferences but also determine when and how to present service by accommodating context information (e.g. location, time, priority, and driver's mood).

\section{Open Issues}

On the basis of the literature review on state-of-the-art technologies for implicit personalized driving assistance, this section further highlights some open issues in personalized driving assistance so as to facilitate its future research.

\section{A. Utilization of Existing Driving Dataset and Personal Data Collection}

Data-driven approaches not only play a significant role in driving assistance but also for the entire Intelligent Transportation Systems [139]. Thanks to the great work in [36, 140], lots of important driving datasets are summarized and described in detail. In this paper, we attempt to supplement more driving datasets along with detailed descriptions and their open access status. Therefore, several existing datasets and their scale, source types, and potential applications are elaborated in this 
TABLE V

Datasets and Potential APPlications

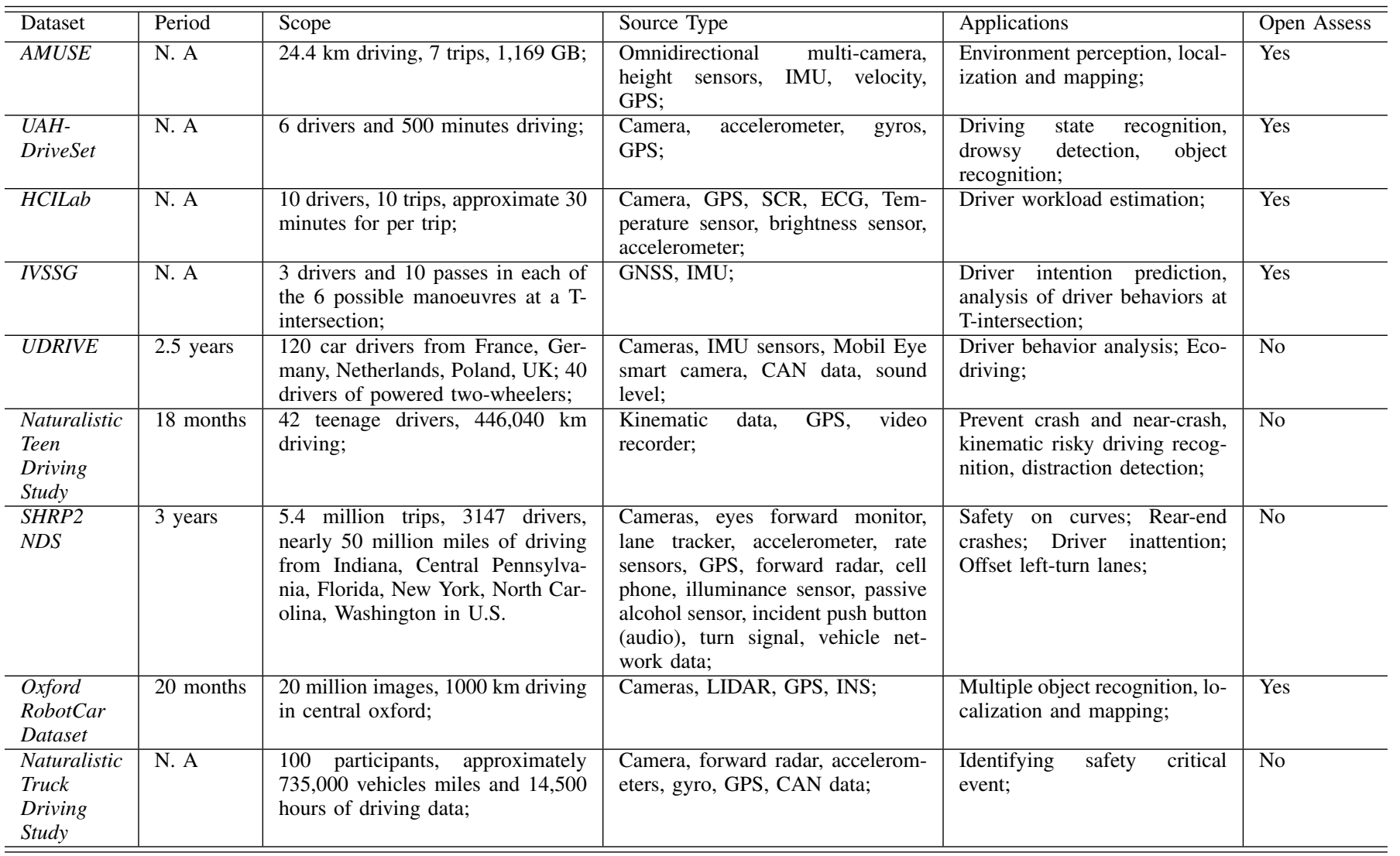

section and summarized in Table V. In particular, AMUSE Dataset consists of inertial and other complementary sensor data combined with monocular, omnidirectional, high frame rate visual data taken in real traffic scenes during multiple test drives [141]. UAH-DriveSet is a publicly available dataset which was collected in 2016 by using a smartphone app DriveSafe for in-depth analysis of driving behaviors [28]. HCILab Dataset is collected to assess driver workload and includes a variety of physiological data, video data, GPS, accelerometer data are measured [142]. IVSSG is collected from a vehicle driving around urban street around the Australian Centre for Field Robotics in Sydney and includes data from a GPS, gyroscopes, and odometers are adopted [143]. UDRIVE is the first large-scale European Naturalistic Driving Study on cars, trucks and powered two-wheelers. The acronym stands for European naturalistic Driving and Riding for Infrastructure \& Vehicle safety and Environment. The purpose of the study is to gain a better understanding of what happens on the road in everyday traffic situations [144]. SHRP2 NDS is a very large-scale follow-up study which is the second Strategic Highway Research Program (SHRP2) [145]. This study involved more than 3000 participants in six sites of U.S. Naturalistic Truck Driving Study fits nine trucks with a suite of sensors. This study recruited 100 drivers from four different trucking fleets across seven terminals for exploring commercial motor vehicle risk by identifying safety-critical events [146]. Oxford RobotCar Dataset is collected by the
Oxford Robotics Institute. The driving data was recorded from May 2014 to December 2015. As a result, 1000 km driving data were collected including image, LIDAR, GPS and INS data [147]. Naturalistic Teenage Driving Study is focused on teenage drivers to explore their risks in driving. The study lasted for 18 months and involved 42 teenage drivers [145].

However, most of the aforementioned datasets do not provide unique IDs to indicate different drivers, which causes difficulties to test personalized driving assistance services. It should be noted that personal data collection is the basis of personalized services. The personalized systems can outperform the average systems when sufficient personal data is available. Until now, most data acquisition systems collect driving data indiscriminately. As a result, personalized driving characteristics and preferences of individual drivers are overlooked when several drivers share a vehicle. Therefore, how to implement personal data collection is an important outstanding problem for personalized driving assistance.

\section{B. Cold-start Problems}

Cold-start problems occur when insufficient personalized data is available for a new user and consist of two categories: cold-start items and cold-start users [148]. In driving assistance applications, the cold-start item problems relate to service recommendations such as route and music recommendations. Cold-start users refer to a fast adaptation of an individual to provide a better driving experience. Cold-start problems are 
significant for driving assistance applications because drivers may abandon the applications if false positive rate is too high during its initial phase.

\section{Personalization in Driver Monitoring Systems}

It is outlined in Section IV that several human factor challenges, such as trust, acceptance, and unpredictability [98, 103, 104], may slow down the development of DMS. For now, not many studies have been conducted on personalized DMS. Most studies in DMS are to build average models, find more relevant indicators or improve performance by developing or using more advanced algorithms. To fill this research gap, more research about personalized driver monitoring systems needs to be done for trustworthy collaboration between human drivers and vehicles.

\section{Personalization for Surrounding Vehicles}

Driving is a cooperative task, where ego-vehicle needs to interact with surrounding vehicles [149]. This requires the ability to make decisions in dynamic and potentially uncertain environments [150]. The uncertainty does not only come from noisy sensor data, but also is due to the fact that human actions and behaviors are very difficult to predict [98]. In order to enhance prediction accuracy, the surrounding vehicles should be personalized (e.g. aggressive driver, conservative driver) so that the intentions of surrounding vehicles can be made more predictable. The problem can be summarized as: (1) what is the most useful indicators? (2) how to predict a driver's intention by only observing her/his driving behaviors for a short period (minutes, even seconds)?

\section{E. Online Unsupervised Personalized Learning Problems}

Personalization is often viewed as a static process. Once a personalized model is constructed, its parameters and construction cannot be tuned or changed any more until the personalized model is completely retrained. In real-life applications, a personalized system needs to be updated and improved continuously by using cues from driver interaction, i.e. online personalized learning systems. This is due to the fact that driving preferences and characteristics may change with time even for the same driver. For instance, driving preferences and characteristics may change from a cautious style to a normal style when drivers accumulate more driving experience. This issue is also highlighted in [42]. However, only achieving online learning is not enough for personalized application. This is due to the fact that manually labeling personal data is laborious and inefficient. To this end, realizing personalization in the online and unsupervised way is a big challenge for personalized driving assistance systems.

\section{F. Social Interactive Assistance}

Another poorly explored aspect is the social interactive assistance between a personalized smart vehicle and a driver. Compared to a conventional human-machine interface design, social interactive assistance is more advanced and more challenging which needs to provide humanized services at the correct context (e.g. time and place) and in the appropriate manner (e.g. mood, audio, and vision). The interaction between vehicles and drivers affects the quality of personalization. A user may make a trade-off between side effects (e.g., high false alarm rate, complex operation) and benefits of personalized systems. This issue is discussed comprehensively in [151].

\section{CONCLUSIONS}

This paper provided an overview of state-of-the-art developments in implicit personalized driving assistance and discussed open issues that still need to be addressed. The previous achievements of personalized driving assistance were investigated in SDS, DMS, and IVIS. Based on this review, some open issues were discovered such as utilization of existing driving dataset and personal data collection, cold-start problems, limited work in personalized DMS, online unsupervised personalized learning, personalization for surrounding vehicles, and personalized social interactive assistance. Additionally, implicit personalized driving assistance was generally implemented by using data-driven approaches which are dataintensive applications. Therefore, we also summarized existing driving datasets and explored their potential applications. It is anticipated that this survey paper would be particularly useful for researchers who are about to enter this exciting area.

To aid drivers with appropriate assistance at the right time, driving assistance systems require a deeper understanding of drivers' behaviors. Data-driven approaches are promising solutions which can process large-scale data and adapt to individual drivers. With more personalized data, future work shall concentrate on mining of big data suggesting that more advanced machine learning algorithms should be applied in formulating personalized preferences and characteristics such as deep reinforcement learning and transfer learning. Another trend shall focus on seamlessly integrating personalized learning algorithms and vehicle control systems. A barrier of popularizing driverless cars is about how to make drivers trust and enjoy driverless cars so as to enhance the riding experience. Personalized driving assistance could provide a promising answer to this question. Personalized driving assistance is not only important to support manual driving but also making fully autonomous driving better for individual needs.

Moreover, this paper is mainly focused on categorizing driving assistance systems according to their application domains, which include SDS (vehicle dynamics and control related functions), DMS (human driver surveillance and forewarning), and IVIS (information provision and interaction). However, driving assistance systems can also be categorized based on automation levels and/or human-vehicle shared control types. This is not covered due to length limitation, but is treated future work for interested researchers.

\section{REFERENCES}

[1] K. Liu, J. Gong, A. Kurt, H. Chen, and U. Ozguner, "Dynamic modeling and control of high-speed automated vehicles for lane change maneuver," IEEE Transactions on Intelligent Vehicles, vol. 3, no. 3, pp. 329-339, 2018. 
[2] D. Yi, J. Su, C. Liu, M. Quddus, and W.-H. Chen, "A machine learning based personalized system for driving state recognition," Transportation Research Part C: Emerging Technologies, vol. 105, pp. 241-261, 2019.

[3] D. Yi, J. Su, C. Liu, and W.-H. Chen, "Personalized driver workload inference by learning from vehicle related measurements," IEEE Transactions on Systems, Man, and Cybernetics: Systems, vol. 49, no. 1, pp. 159-168, 2019.

[4] E. Ohn-Bar and M. M. Trivedi, "Looking at humans in the age of self-driving and highly automated vehicles," IEEE Transactions on Intelligent Vehicles, vol. 1, no. 1, pp. 90-104, 2016.

[5] D. Yi, J. Su, C. Liu, and W.-H. Chen, "New driver workload prediction using clustering-aided approaches," IEEE Transactions on Systems, Man, and Cybernetics: Systems, vol. 49, no. 1, pp. 64-70, 2019.

[6] B. Paden, M. Čáp, S. Z. Yong, D. Yershov, and E. Frazzoli, "A survey of motion planning and control techniques for self-driving urban vehicles," IEEE Transactions on intelligent vehicles, vol. 1, no. 1, pp. 33-55, 2016.

[7] D. Bevly, X. Cao, M. Gordon, G. Ozbilgin, D. Kari, B. Nelson, J. Woodruff, M. Barth, C. Murray, A. Kurt et al., "Lane change and merge maneuvers for connected and automated vehicles: A survey," IEEE Transactions on Intelligent Vehicles, vol. 1, no. 1, pp. 105-120, 2016.

[8] S. Kaplan, M. A. Guvensan, A. G. Yavuz, and Y. Karalurt, "Driver behavior analysis for safe driving: a survey," IEEE Transactions on Intelligent Transportation Systems, vol. 16, no. 6, pp. 3017-3032, 2015.

[9] S. Nanga, N. A. Odai, and A. Lotsi, "Survival pattern of first accident among commercial drivers in the greater accra region of ghana," Accident Analysis \& Prevention, vol. 103, pp. 9295, 2017.

[10] W. Balid, H. Tafish, and H. H. Refai, "Intelligent vehicle counting and classification sensor for real-time traffic surveillance," IEEE Transactions on Intelligent Transportation Systems, vol. 19, no. 6, pp. 1784-1794, 2018.

[11] M. Popken, A. Rosenow, and M. Lübcke, "Driver assistance systems," ATZextra worldwide, vol. 12, no. 1, pp. 210-215, 2007.

[12] R. Katzwinkel and S. Kopischke, "Driver assistance systems," ATZextra worldwide, vol. 14, no. 12, pp. 48-55, 2009.

[13] A. Vukotich, R. G. Salvador, A. M. Martínez, and H. Töpper, "Driver assistance systems," ATZextra worldwide, vol. 16, no. 7, pp. 86-95, 2011.

[14] Z. Darowich, "Driver monitoring system," Jun. 23 2016, uS Patent App. 14/573,266.

[15] T. Wynn, J. H. Richardson, and A. Stevens, "Driving whilst using in-vehicle information systems (ivis): benchmarking the impairment to alcohol." (c) Ashgate Publishing, 2013.

[16] W. Wang, W. Zhang, and D. Zhao, "Understanding V2V Driving Scenarios through Traffic Primitives," arXiv preprint arXiv:1807.10422, 2018.

[17] W. Wang, J. Xi, and H. Chen, "Modeling and recognizing driver behavior based on driving data: A survey," Mathematical Problems in Engineering, vol. 2014, 2014.

[18] V. A. Butakov and P. A. Ioannou, "Driver/vehicle response diagnostic system for the vehicle-following case," IEEE Transactions on Intelligent Transportation Systems, vol. 15, no. 5, pp. 1947-1957, 2014.

[19] Y. Nishiwaki, C. Miyajima, N. Kitaoka, K. Itou, and K. Takeda, "Generation of pedal operation patterns of individual drivers in car-following for personalized cruise control," in IEEE Intelligent Vehicles Symposium (IV), 2007, pp. 823-827.

[20] J. Wang, L. Zhang, D. Zhang, and K. Li, "An adaptive longitudinal driving assistance system based on driver characteristics," IEEE Transactions on Intelligent Transportation Systems, vol. 14, no. 1, pp. 1-12, 2013.

[21] C. Miyajima, Y. Nishiwaki, K. Ozawa, T. Wakita, K. Itou,
K. Takeda, and F. Itakura, "Driver modeling based on driving behavior and its evaluation in driver identification," Proceedings of the IEEE, vol. 95, no. 2, pp. 427-437, 2007.

[22] C. M. Martinez, M. Heucke, F.-Y. Wang, B. Gao, and D. Cao, "Driving style recognition for intelligent vehicle control and advanced driver assistance: A survey," IEEE Transactions on Intelligent Transportation Systems, vol. 19, no. 3, pp. 666-676, 2018.

[23] K. Inata, P. Raksincharoensak, and M. Nagai, "Driver behavior modeling based on database of personal mobility driving in urban area," in IEEE International Conference on Control, Automation and Systems, 2008, pp. 2902-2907.

[24] Y. Sun and X. B. Yu, "An innovative nonintrusive driver assistance system for vital signal monitoring," IEEE journal of biomedical and health informatics, vol. 18, no. 6, pp. 19321939, 2014.

[25] M.-H. Park, J.-H. Hong, and S.-B. Cho, "Location-based recommendation system using bayesian users preference model in mobile devices," in International Conference on Ubiquitous Intelligence and Computing. Springer, 2007, pp. 1130-1139.

[26] W. Wang, J. Xi, and D. Zhao, "Learning and inferring a driver's braking action in car-following scenarios," IEEE Transactions on Vehicular Technology, vol. 67, no. 5, pp. 3887-3899, 2018.

[27] S. Schnelle, J. Wang, H. Su, and R. Jagacinski, "A driver steering model with personalized desired path generation," IEEE Transactions on Systems, Man, and Cybernetics: Systems, vol. 47, no. 1, pp. 111-120, 2017.

[28] E. Romera, L. M. Bergasa, and R. Arroyo, "Need data for driver behaviour analysis? Presenting the public UAHDriveSet," in Proc. IEEE 19th Int. Conf. Intell. Transp. Syst. (ITSC). Rio de Janeiro, Brazil, 2016, pp. 387-392.

[29] V. A. Butakov and P. Ioannou, "Personalized driver/vehicle lane change models for adas," IEEE Transactions on Vehicular Technology, vol. 64, no. 10, pp. 4422-4431, 2015.

[30] F.-Y. Wang, D. Zeng, and L. Yang, "Smart cars on smart roads: an ieee intelligent transportation systems society update," IEEE Pervasive Computing, vol. 5, no. 4, pp. 68-69, 2006.

[31] D. Yi, J. Su, C. Liu, and W.-H. Chen, "Trajectory clustering aided personalized driver intention prediction for intelligent vehicles," IEEE Transactions on Industrial Informatics, 2018.

[32] T. Wilhelem, H. Okuda, B. Levedahl, and T. Suzuki, "Energy consumption evaluation based on a personalized driver-vehicle model," IEEE Transactions on Intelligent Transportation Systems, vol. 18, no. 6, pp. 1468-1477, 2017.

[33] C. Chen, D. Zhang, B. Guo, X. Ma, G. Pan, and Z. Wu, "Tripplanner: Personalized trip planning leveraging heterogeneous crowdsourced digital footprints," IEEE Transactions on Intelligent Transportation Systems, vol. 16, no. 3, pp. 1259$1273,2015$.

[34] S. M. Simmons, J. K. Caird, and P. Steel, "A meta-analysis of in-vehicle and nomadic voice-recognition system interaction and driving performance," Accident Analysis \& Prevention, vol. 106, pp. 31-43, 2017.

[35] V. Butakov, "Personalized driver assistance systems based on driver/vehicle models," Ph.D. dissertation, University of Southern California, 2014.

[36] W. Wang, C. Liu, and D. Zhao, "How much data are enough? A statistical approach with case study on longitudinal driving behavior," IEEE Transactions on Intelligent Vehicles, vol. 2, no. 2, pp. 85-98, 2017.

[37] M. Kaptein, P. Markopoulos, B. De Ruyter, and E. Aarts, "Personalizing persuasive technologies: Explicit and implicit personalization using persuasion profiles," International Journal of Human-Computer Studies, vol. 77, pp. 38-51, 2015.

[38] J. Teevan, S. T. Dumais, and E. Horvitz, "Potential for personalization," ACM Transactions on Computer-Human Interaction (TOCHI), vol. 17, no. 1, p. 4, 2010.

[39] M. Treiber, A. Hennecke, and D. Helbing, "Congested traffic states in empirical observations and microscopic simulations,", 
Physical review E, vol. 62, no. 2, p. 1805, 2000.

[40] L. M. Bergasa, J. Nuevo, M. A. Sotelo, R. Barea, and M. E. Lopez, "Real-time system for monitoring driver vigilance," IEEE Transactions on Intelligent Transportation Systems, vol. 7, no. 1 , pp. 63-77, 2006.

[41] Q. Ji, Z. Zhu, and P. Lan, "Real-time nonintrusive monitoring and prediction of driver fatigue," IEEE transactions on vehicular technology, vol. 53, no. 4, pp. 1052-1068, 2004.

[42] M. Hasenjäger and H. Wersing, "Personalization in advanced driver assistance systems and autonomous vehicles: A review," in 2017 IEEE 20th International Conference on Intelligent Transportation Systems (ITSC). IEEE, 2017, pp. 1-7.

[43] H. Okuda, N. Ikami, T. Suzuki, Y. Tazaki, and K. Takeda, "Modeling and analysis of driving behavior based on a probability-weighted arx model," IEEE Transactions on Intelligent Transportation Systems, vol. 14, no. 1, pp. 98-112, 2013.

[44] Y. Jin, W. Von Seelen, and B. Sendhoff, "On generating fc/sup 3/fuzzy rule systems from data using evolution strategies," IEEE Transactions on Systems, Man, and Cybernetics, Part B (Cybernetics), vol. 29, no. 6, pp. 829-845, 1999.

[45] Y. Jin and J. Branke, "Evolutionary optimization in uncertain environments-a survey," IEEE Transactions on evolutionary computation, vol. 9, no. 3, pp. 303-317, 2005.

[46] R. Isermann, "On fuzzy logic applications for automatic control, supervision, and fault diagnosis," IEEE Transactions on Systems, Man, and Cybernetics-Part A: Systems and Humans, vol. 28, no. 2, pp. 221-235, 1998.

[47] G. N. Bifulco, F. Simonelli, and R. Di Pace, "Experiments toward an human-like adaptive cruise control," in Intelligent Vehicles Symposium, 2008 IEEE. IEEE, 2008, pp. 919-924.

[48] G. P. Zhang, "Time series forecasting using a hybrid arima and neural network model," Neurocomputing, vol. 50, pp. 159-175, 2003.

[49] J. V. Tu, "Advantages and disadvantages of using artificial neural networks versus logistic regression for predicting medical outcomes," Journal of clinical epidemiology, vol. 49, no. 11, pp. 1225-1231, 1996.

[50] D. F. Specht, "A general regression neural network," IEEE transactions on neural networks, vol. 2, no. 6, pp. 568-576, 1991.

[51] G. N. Bifulco, L. Pariota, F. Simonelli, and R. Di Pace, "Development and testing of a fully adaptive cruise control system," Transportation Research Part C: Emerging Technologies, vol. 29, pp. 156-170, 2013.

[52] H. Permuter, J. Francos, and I. Jermyn, "A study of gaussian mixture models of color and texture features for image classification and segmentation," Pattern Recognition, vol. 39, no. 4, pp. 695-706, 2006.

[53] S. Lefèvre, A. Carvalho, and F. Borrelli, "A learning-based framework for velocity control in autonomous driving," IEEE Transactions on Automation Science and Engineering, vol. 13, no. 1, pp. 32-42, 2016.

[54] J. Yamato, J. Ohya, and K. Ishii, "Recognizing human action in time-sequential images using hidden markov model," in in Proc. IEEE Conf. Comput. Vis. Pattern Recognit., 1992, pp. 379-385.

[55] P. Smyth, "Hidden markov models for fault detection in dynamic systems," Pattern recognition, vol. 27, no. 1, pp. 149$164,1994$.

[56] S. R. Eddy, "Profile hidden markov models." Bioinformatics (Oxford, England), vol. 14, no. 9, pp. 755-763, 1998.

[57] S. Lefèvre, A. Carvalho, Y. Gao, H. E. Tseng, and F. Borrelli, "Driver models for personalised driving assistance," Vehicle System Dynamics, vol. 53, no. 12, pp. 1705-1720, 2015.

[58] S. Ramyar, A. Homaifar, S. M. Salaken, S. Nahavandi, and A. Kurt, "A personalized highway driving assistance system," in Proc. IEEE IV Symp., 2017, pp. 1596-1601.

[59] L. Breiman, "Random forests," Machine learning, vol. 45, no. 1, pp. 5-32, 2001.
[60] M. Pal, "Random forest classifier for remote sensing classification," International Journal of Remote Sensing, vol. 26, no. 1, pp. 217-222, 2005.

[61] P. Baudron, F. Alonso-Sarría, J. L. García-Aróstegui, F. Cánovas-García, D. Martínez-Vicente, and J. MorenoBrotóns, "Identifying the origin of groundwater samples in a multi-layer aquifer system with random forest classification," Journal of Hydrology, vol. 499, pp. 303-315, 2013.

[62] S. Ren, X. Cao, Y. Wei, and J. Sun, "Global refinement of random forest," in Proceedings of the IEEE Conference on Computer Vision and Pattern Recognition, 2015, pp. 723-730.

[63] Y. Chen, T. Le-Ngoc, B. Champagne, and C. Xu, "Recursive least squares constant modulus algorithm for blind adaptive array," IEEE Transactions on Signal Processing, vol. 52, no. 5, pp. 1452-1456, 2004.

[64] F. Ling, D. Manolakis, and J. Proakis, "A recursive modified gram-schmidt algorithm for least-squares estimation," IEEE transactions on acoustics, speech, and signal processing, vol. 34, no. 4, pp. 829-836, 1986.

[65] V. Butakov, P. Ioannou, M. Tippelhofer, and J. Camhi, "Driver/vehicle response diagnostic system for vehicle following based on gaussian mixture model," in Decision and Control (CDC), 2012 IEEE 51st Annual Conference on. IEEE, 2012, pp. 5649-5654.

[66] A. P. Bolduc, L. Guo, and Y. Jia, "Multimodel approach to personalized autonomous adaptive cruise control," IEEE Transactions on Intelligent Vehicles, vol. 4, no. 2, pp. 321330, 2019.

[67] F. Muehlfeld, I. Doric, R. Ertlmeier, and T. Brandmeier, "Statistical behavior modeling for driver-adaptive precrash systems," IEEE Transactions on Intelligent Transportation Systems, vol. 14, no. 4, pp. 1764-1772, 2013.

[68] J. Wang, C. Yu, S. E. Li, and L. Wang, "A forward collision warning algorithm with adaptation to driver behaviors," IEEE Transactions on Intelligent Transportation Systems, vol. 17, no. 4, pp. 1157-1167, 2016.

[69] Q. Huo and B. Ma, "Online adaptive learning of continuousdensity hidden markov models based on multiple-stream prior evolution and posterior pooling," IEEE transactions on speech and audio processing, vol. 9, no. 4, pp. 388-398, 2001.

[70] N. Noto, H. Okuda, Y. Tazaki, S. Inagaki, and T. Suzuki, "Obstacle avoidance assisting system based on personalized potential field," in SICE Annual Conference (SICE), 2011 Proceedings of. IEEE, 2011, pp. 476-481.

[71] Y. K. Hwang and N. Ahuja, "A potential field approach to path planning," IEEE Transactions on Robotics and Automation, vol. 8, no. 1, pp. 23-32, 1992.

[72] Y. Koren and J. Borenstein, "Potential field methods and their inherent limitations for mobile robot navigation," in Robotics and Automation, 1991. Proceedings., 1991 IEEE International Conference on. IEEE, 1991, pp. 1398-1404.

[73] H. Okuda, S. Yoshino, T. Suzuki, and T. Kawai, "Identification of mode switching condition in overtaking behavior using variable-free logistic regression model," in 2015 IEEE 18th International Conference on Intelligent Transportation Systems. IEEE, 2015, pp. 1539-1544.

[74] G. Li, S. E. Li, Y. Liao, W. Wang, B. Cheng, and F. Chen, "Lane change maneuver recognition via vehicle state and driver operation signalsresults from naturalistic driving data," in Proc. IEEE IV Symp., 2015, pp. 865-870.

[75] M. Hasenjäger and $\mathrm{H}$. Wersing, "Personalization in advanced driver assistance systems and autonomous vehicles: A review."

[76] S. Patil, J. Van Den Berg, and R. Alterovitz, "Estimating probability of collision for safe motion planning under Gaussian motion and sensing uncertainty," in 2012 IEEE International Conference on Robotics and Automation. IEEE, 2012, pp. 3238-3244.

[77] W. Wang, D. Zhao, W. Han, and J. Xi, “A learning-based approach for lane departure warning systems with a personalized 
driver model," IEEE Transactions on Vehicular Technology, vol. 67, no. 10, pp. 9145-9157, 2018.

[78] H. Okuda, K. Harada, T. Suzuki, S. Saigo, and S. Inoue, "Design of automated merging control by minimizing decision entropy of drivers on main lane," in 2017 IEEE Intelligent Vehicles Symposium (IV). IEEE, 2017, pp. 640-646.

[79] Y. Suehiro, T. Wada, and K. Sonoda, "A driver assistance method for merging to increase clearness of driver decision making," in 2017 IEEE/SICE International Symposium on System Integration (SII). IEEE, 2017, pp. 559-564.

[80] W. Wang, J. Xi, C. Liu, and X. Li, "Human-Centered FeedForward Control of a Vehicle Steering System Based on a Driver's Path-Following Characteristics," IEEE Transactions on Intelligent Transportation Systems, vol. 18, no. 6, pp. 1440 1453, 2017.

[81] Y. Shan and K. K. Leang, "Frequency-weighted feedforward control for dynamic compensation in ionic polymer-metal composite actuators," Smart materials and structures, vol. 18, no. 12, p. 125016, 2009.

[82] L. Bi, Y. Lu, X. Fan, J. Lian, and Y. Liu, "Queuing network modeling of driver eeg signals-based steering control," IEEE Transactions on Neural Systems and Rehabilitation Engineering, vol. 25, no. 8, pp. 1117-1124, 2017.

[83] J. M. Smith and D. Towsley, "The use of queuing networks in the evaluation of egress from buildings," Environment and Planning B: Planning and Design, vol. 8, no. 2, pp. 125-139, 1981.

[84] C. H. Sauer, E. A. MacNair, and S. Salza, "A language for extended queueing network models," IBM Journal of Research and Development, vol. 24, no. 6, pp. 747-755, 1980.

[85] H. U. Yoon, R. F. Wang, and S. A. Hutchinson, "Modeling user's driving-characteristics in a steering task to customize a virtual fixture based on task-performance," in Robotics and Automation (ICRA), 2014 IEEE International Conference on. IEEE, 2014, pp. 625-630.

[86] M. Krstic and Z.-H. Li, "Inverse optimal design of input-tostate stabilizing nonlinear controllers," IEEE Transactions on Automatic Control, vol. 43, no. 3, pp. 336-350, 1998.

[87] F. Ornelas-Tellez, E. N. Sanchez, A. G. Loukianov, and E. M. Navarro-López, "Speed-gradient inverse optimal control for discrete-time nonlinear systems," in Decision and Control and European Control Conference (CDC-ECC), 2011 50th IEEE Conference on. IEEE, 2011, pp. 290-295.

[88] D. Yi, J. Su, C. Liu, and W.-H. Chen, "Data-driven situation awareness algorithm for vehicle lane change," in Proc. IEEE 19th Int. Conf. Intell. Transp. Syst. (ITSC). Rio de Janeiro, Brazil, 2016, pp. 998-1003.

[89] V. A. Butakov and P. Ioannou, "Personalized driver assistance for signalized intersections using v2i communication," IEEE Transactions on Intelligent Transportation Systems, vol. 17, no. 7, pp. 1910-1919, 2016.

[90] T. A. Johansen, T. I. Fossen, and S. P. Berge, "Constrained nonlinear control allocation with singularity avoidance using sequential quadratic programming," IEEE Transactions on Control Systems Technology, vol. 12, no. 1, pp. 211-216, 2004.

[91] D. Orth, D. Kolossa, M. S. Paja, K. Schaller, A. Pech, and M. Heckmann, "A maximum likelihood method for driverspecific critical-gap estimation," in Intelligent Vehicles Symposium (IV), 2017 IEEE. IEEE, 2017, pp. 553-558.

[92] A. H. Eichelberger and A. T. McCartt, "Toyota drivers' experiences with dynamic radar cruise control, pre-collision system, and lane-keeping assist," Journal of safety research, vol. 56, pp. 67-73, 2016.

[93] W. Wang, J. Xi, and J. Wang, "Human-centered feed-forward control of a vehicle steering system based on a driver's steering model," in American Control Conference (ACC), 2015. IEEE, 2015, pp. 3361-3366.

[94] W. Brilon, R. Koenig, and R. J. Troutbeck, "Useful estimation procedures for critical gaps," Transportation Research Part A:
Policy and Practice, vol. 33, no. 3-4, pp. 161-186, 1999.

[95] R. Troutbeck, "Revised raffs method for estimating critical gaps," Transportation research record, vol. 2553, no. 1, pp. $1-9,2016$.

[96] J. M. Wang, D. J. Fleet, and A. Hertzmann, "Gaussian process dynamical models for human motion," IEEE transactions on pattern analysis and machine intelligence, vol. 30, no. 2, pp. 283-298, 2007.

[97] A. Paul, R. Chauhan, R. Srivastava, and M. Baruah, "Advanced driver assistance systems," SAE Technical Paper, Tech. Rep., 2016.

[98] K. Driggs-Campbell, R. Dong, and R. Bajcsy, "Robust, informative human-in-the-loop predictions via empirical reachable sets," IEEE Transactions on Intelligent Vehicles, vol. 3, no. 3, pp. 300-309, 2018

[99] K. Driggs-Campbell and R. Bajcsy, "Identifying modes of intent from driver behaviors in dynamic environments," in 2015 IEEE 18th International Conference on Intelligent Transportation Systems. IEEE, 2015, pp. 739-744.

[100] W. Wang and D. Zhao, "Evaluation of lane departure correction systems using a regenerative stochastic driver model," IEEE Transactions on Intelligent Vehicles, vol. 2, no. 3, pp. 221-232, 2017.

[101] M. Walter, B. Eilebrecht, T. Wartzek, and S. Leonhardt, "The smart car seat: personalized monitoring of vital signs in automotive applications," Personal and Ubiquitous Computing, vol. 15, no. 7, pp. 707-715, 2011.

[102] C. Bila, F. Sivrikaya, M. A. Khan, and S. Albayrak, "Vehicles of the future: A survey of research on safety issues," IEEE Transactions on Intelligent Transportation Systems, vol. 18, no. 5, pp. 1046-1065, 2017.

[103] V. Govindarajan, K. Driggs-Campbell, and R. Bajcsy, "Affective driver state monitoring for personalized, adaptive adas," in 201821 st International Conference on Intelligent Transportation Systems (ITSC). IEEE, 2018, pp. 1017-1022.

[104] K. Driggs-Campbell, V. Shia, and R. Bajcsy, "Improved driver modeling for human-in-the-loop vehicular control," in 2015 IEEE International Conference on Robotics and Automation (ICRA). IEEE, 2015, pp. 1654-1661.

[105] K. Li, M. Lu, F. Lu, Q. Lv, L. Shang, and D. Maksimovic, "Personalized driving behavior monitoring and analysis for emerging hybrid vehicles," in International Conference on Pervasive Computing. Springer, 2012, pp. 1-19.

[106] M. Patel, S. K. Lal, D. Kavanagh, and P. Rossiter, “Applying neural network analysis on heart rate variability data to assess driver fatigue," Expert systems with Applications, vol. 38, no. 6, pp. 7235-7242, 2011.

[107] K. A. Brookhuis and D. de Waard, "Monitoring drivers mental workload in driving simulators using physiological measures," Accident Analysis \& Prevention, vol. 42, no. 3, pp. 898-903, 2010.

[108] Z. Li, S. Bao, I. V. Kolmanovsky, and X. Yin, "Visual-manual distraction detection using driving performance indicators with naturalistic driving data," IEEE Transactions on Intelligent Transportation Systems, vol. 19, no. 8, pp. 2528-2535, 2018.

[109] L. Xu, S. Li, K. Bian, T. Zhao, and W. Yan, "Sober-drive: A smartphone-assisted drowsy driving detection system," in Computing, Networking and Communications (ICNC), 2014 International Conference on. IEEE, 2014, pp. 398-402.

[110] V. Podgorelec, P. Kokol, B. Stiglic, and I. Rozman, "Decision trees: an overview and their use in medicine," Journal of medical systems, vol. 26, no. 5, pp. 445-463, 2002.

[111] S. Dreiseitl and L. Ohno-Machado, "Logistic regression and artificial neural network classification models: a methodology review," Journal of biomedical informatics, vol. 35, no. 5-6, pp. 352-359, 2002.

[112] C.-M. Tseng, C.-K. Chau, S. Dsouza, and E. Wilhelm, "A participatory sensing approach for personalized distance-toempty prediction and green telematics," in Proceedings of the 
2015 ACM Sixth International Conference on Future Energy Systems. ACM, 2015, pp. 47-56.

[113] A. Wahab, C. Quek, C. K. Tan, and K. Takeda, "Driving profile modeling and recognition based on soft computing approach," IEEE transactions on neural networks, vol. 20, no. 4, pp. 563582, 2009.

[114] B. HomChaudhuri and P. Pisu, "A driver-in-the loop fuel economic control strategy for connected vehicles in urban roads," arXiv preprint arXiv:1705.07207, 2017.

[115] S. Di Cairano, D. Bernardini, A. Bemporad, and I. V. Kolmanovsky, "Stochastic mpc with learning for driver-predictive vehicle control and its application to hev energy management," IEEE Transactions on Control Systems Technology, vol. 22, no. 3, pp. 1018-1031, 2014.

[116] G. Ripaccioli, D. Bernardini, S. Di Cairano, A. Bemporad, and I. Kolmanovsky, "A stochastic model predictive control approach for series hybrid electric vehicle power management," in American Control Conference (ACC), 2010. IEEE, 2010, pp. 5844-5849.

[117] L. Blackmore, M. Ono, A. Bektassov, and B. C. Williams, "A probabilistic particle-control approximation of chanceconstrained stochastic predictive control," IEEE transactions on Robotics, vol. 26, no. 3, pp. 502-517, 2010.

[118] C.-M. Tseng and C.-K. Chau, "Personalized prediction of vehicle energy consumption based on participatory sensing," IEEE Transactions on Intelligent Transportation Systems, 2017.

[119] Y. Shi, M. Larson, and A. Hanjalic, "List-wise learning to rank with matrix factorization for collaborative filtering," in Proceedings of the fourth ACM conference on Recommender systems. ACM, 2010, pp. 269-272.

[120] S. Rendle and L. Schmidt-Thieme, "Online-updating regularized kernel matrix factorization models for large-scale recommender systems," in Proceedings of the 2008 ACM conference on Recommender systems. ACM, 2008, pp. 251-258.

[121] K. Yu, A. Schwaighofer, V. Tresp, X. Xu, and H.-P. Kriegel, "Probabilistic memory-based collaborative filtering," IEEE Transactions on Knowledge and Data Engineering, vol. 16, no. 1, pp. 56-69, 2004.

[122] B. Shi, L. Xu, J. Hu, Y. Tang, H. Jiang, W. Meng, and H. Liu, "Evaluating driving styles by normalizing driving behavior based on personalized driver modeling," IEEE Transactions on Systems, Man, and Cybernetics: Systems, vol. 45, no. 12, pp. 1502-1508, 2015.

[123] Y. Dong, Z. Hu, K. Uchimura, and N. Murayama, "Driver inattention monitoring system for intelligent vehicles: A review," IEEE transactions on intelligent transportation systems, vol. 12, no. 2, pp. 596-614, 2011.

[124] J. Yuan, Y. Zheng, X. Xie, and G. Sun, "T-drive: Enhancing driving directions with taxi drivers' intelligence," IEEE Transactions on Knowledge and Data Engineering, vol. 25, no. 1, pp. 220-232, 2013.

[125] L. Mc Ginty and B. Smyth, "Collaborative case-based reasoning: Applications in personalised route planning," in International Conference on Case-Based Reasoning. Springer, 2001, pp. 362-376.

[126] P. Ioannou and Y. Zhang, "Intelligent driver assist system for urban driving," in Digital Media Industry \& Academic Forum (DMIAF). IEEE, 2016, pp. 128-134.

[127] N. Mahajan, A. Hegyi, S. P. Hoogendoorn, and B. van Arem, "Design analysis of a decentralized equilibrium-routing strategy for intelligent vehicles," Transportation Research Part C: Emerging Technologies, vol. 103, pp. 308-327, 2019.

[128] S. R. Garzon, "Intelligent in-car-infotainment systems: A contextual personalized approach," in IEEE 8th International Conference on Intelligent Environments (IE), 2012, pp. 315318.

[129] S. Goose, L. Riddle, C. Fuller, T. Gupta, and A. Marcus, "Paz: In-vehicle personalized audio zones," IEEE MultiMedia, vol. 23 , no. 4, pp. 32-41, 2016.
[130] A. P. Chanda, A. Deb, A. Kar, and M. Chandra, "An improved filtered-x least mean square algorithm for cancellation of single-tone and multitone noise," in Medical Imaging, $m$ Health and Emerging Communication Systems (MedCom), 2014 International Conference on. IEEE, 2014, pp. 358-363.

[131] J. I. Árnason, J. Jepsen, A. Koudal, M. R. Schmidt, and S. Serafin, "Volvo intelligent news: A context aware multi modal proactive recommender system for in-vehicle use," Pervasive and Mobile Computing, vol. 14, pp. 95-111, 2014.

[132] H. Oh, L. Jalali, and R. Jain, "An intelligent notification system using context from real-time personal activity monitoring," in IEEE International Conference on Multimedia and Expo (ICME), 2015, pp. 1-6.

[133] Y. Peng and L. N. Boyle, "Driver's adaptive glance behavior to in-vehicle information systems," Accident Analysis \& Prevention, vol. 85, pp. 93-101, 2015.

[134] P. Clarke, C. Crawford, F. Steele, and A. F. Vignoles, "The choice between fixed and random effects models: some considerations for educational research," 2010.

[135] M. M. Moniri, M. Feld, and C. Müller, "Personalized invehicle information systems: Building an application infrastructure for smart cars in smart spaces," in Intelligent Environments (IE), 2012 8th International Conference on. IEEE, 2012, pp. 379-382.

[136] K. J. Williams, J. C. Peters, and C. L. Breazeal, "Towards leveraging the driver's mobile device for an intelligent, sociable in-car robotic assistant," in Intelligent Vehicles Symposium (IV), 2013 IEEE. IEEE, 2013, pp. 369-376.

[137] K. Williams, J. A. Flores, and J. Peters, "Affective robot influence on driver adherence to safety, cognitive load reduction and sociability," in Proceedings of the 6th International Conference on Automotive User Interfaces and Interactive Vehicular Applications. ACM, 2014, pp. 1-8.

[138] M. Braun, N. Broy, B. Pfleging, and F. Alt, "Visualizing natural language interaction for conversational in-vehicle information systems to minimize driver distraction," Journal on Multimodal User Interfaces, pp. 1-18, 2019.

[139] J. Zhang, F.-Y. Wang, K. Wang, W.-H. Lin, X. Xu, and C. Chen, "Data-driven intelligent transportation systems: A survey," IEEE Transactions on Intelligent Transportation Systems, vol. 12, no. 4, pp. 1624-1639, 2011.

[140] W. Wang and D. Zhao, "Extracting traffic primitives directly from naturalistically logged data for self-driving applications," IEEE Robotics and Automation Letters, vol. 3, no. 2, pp. 12231229, 2018.

[141] P. Koschorrek, T. Piccini, P. Oberg, M. Felsberg, L. Nielsen, and R. Mester, "A multi-sensor traffic scene dataset with omnidirectional video," in in Proc. IEEE Conf. Comput. Vis. Pattern. Recognit., 2013, pp. 727-734.

[142] S. Schneegass, B. Pfleging, N. Broy, F. Heinrich, and A. Schmidt, "A data set of real world driving to assess driver workload," in Proceedings of the 5th international conference on automotive user interfaces and interactive vehicular applications. ACM, 2013, pp. 150-157.

[143] A. Bender, J. R. Ward, S. Worrall, and E. M. Nebot, "Predicting driver intent from models of naturalistic driving," in Intelligent Transportation Systems (ITSC), 2015 IEEE 18th International Conference on. IEEE, 2015, pp. 1609-1615.

[144] Y. Barnard, F. Utesch, N. van Nes, R. Eenink, and M. Baumann, "The study design of udrive: the naturalistic driving study across europe for cars, trucks and scooters," European Transport Research Review, vol. 8, no. 2, p. 14, 2016.

[145] B. G. Simons-Morton, S. G. Klauer, M. C. Ouimet, F. Guo, P. S. Albert, S. E. Lee, J. P. Ehsani, A. K. Pradhan, and T. A. Dingus, "Naturalistic teenage driving study: Findings and lessons learned," Journal of safety research, vol. 54, pp. 41-48, 2015.

[146] R. Olson, R. Bocanegra et al., "Driver distraction in commercial vehicle operations. blacksburg va," Center for Truck 
and Bus Safety, Virginia Tech Transportation Institute, p. 285, 2009.

[147] W. Maddern, G. Pascoe, C. Linegar, and P. Newman, "1 year, 1000 km: The oxford robotcar dataset." IJ Robotics Res., vol. 36, no. 1, pp. 3-15, 2017.

[148] D. Adomavicius and A. Tuzhilin, "Personalization technologies: A process-oriented perspective," Wirtschaftsinformatik, vol. 48, no. 6, pp. 449-450, 2006.

[149] C. Hubmann, M. Becker, D. Althoff, D. Lenz, and C. Stiller, "Decision making for autonomous driving considering interaction and uncertain prediction of surrounding vehicles," in Proc. IEEE IV Symp. IEEE, 2017, pp. 1671-1678.

[150] C. Hubmann, J. Schulz, M. Becker, D. Althoff, and C. Stiller, "Automated driving in uncertain environments: Planning with interaction and uncertain maneuver prediction," IEEE Transactions on Intelligent Vehicles, vol. 3, no. 1, pp. 5-17, 2018.

[151] A. D. Jameson, "Understanding and dealing with usability side effects of intelligent processing," AI Magazine, vol. 30, no. 4, p. 23, 2009.

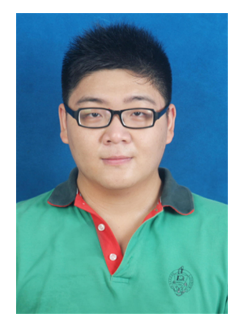

Dewei Yi (S'16, M'18) received his B.Eng. degree in 2014 in Software Engineering from Zhejiang University of Technology, Zhejiang, China. In 2015, he obtained his M.Sc. degree from the Department of Computer Science, Loughborough University, Loughborough, U.K. In 2018, he received a Ph.D degree from the Department of Aeronautical and Automotive Engineering, Loughborough University, Loughborough, U.K. Since 2019, he is a research fellow with the Warwick Manufacturing Group (WMG), University of Warwick, Coventry, U.K. His current research interests include personalized driving assistance, autonomous vehicle and vehicular network.

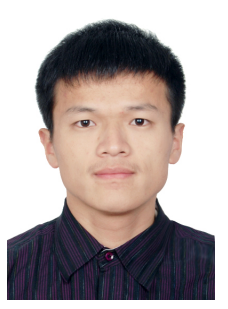

Jinya Su (S'13, M'16) received his B.Sc. degree in Mathematics from Shandong University, China in 2011. In 2016, he received a Ph.D degree in the Department of Aeronautical and Automotive Engineering, Loughborough University, U.K. From 2015, he was a research associate in the same institute. He joined the School of Computer Science and Electronic Engineering, University of Essex, as a lecturer in Computer Science and AI in 2019. His research interests include Kalman filter, machine learning and their applications to autonomous systems such as intelligent vehicle, agricultural information system.

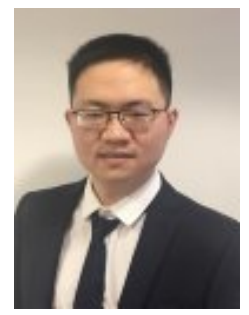

Liang Hu (M'18) is a Lecturer in the School of Computer Science and Electronic Engineering at University of Essex, UK. He received both the BEng and MEng degrees from Harbin Institute of Technology, China, in 2008 and 2010, respectively, and the PhD degree in Computer Science from Brunel University London, UK, in 2016. Prior to joining University of Essex, he was a postdoc researcher at Queen's University Belfast, Loughborough University from 2016 to 2018, and a Lecturer at De Montfort University, U.K. from 2018 to 2019. Dr Hu's research focuses on control and signal processing and their applications in intelligent autonomous systems and cyber-physical systems.

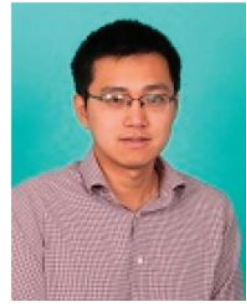

Cunjia Liu received his B.Eng. and M.Sc. degrees in guidance, navigation, and control from Beihang University, Beijing, China, in 2005 and 2008. In 2011, he received a Ph.D. degree in autonomous vehicle control from Loughborough University, Loughborough, United Kingdom. From 2011, he was a research associate in the same institute, where he was appointed as a lecturer in flight dynamics and control in 2013 and a Senior Lecturer in 2018. His current research interests include optimizationbased control, disturbance-observer based control, Bayesian information fusion, and their applications to autonomous vehicles for flight control, path planning, decision making, and situation awareness.

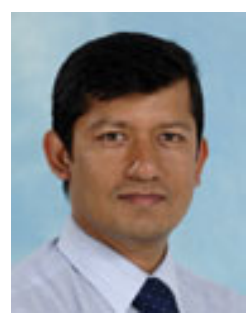

Mohammed Quddus received the bachelors degree in civil engineering from Bangladesh University of Engineering and Technology in 1998, the masters degree in transportation engineering from National University of Singapore in 2001, and the Ph.D. degree in intelligent transportation systems from Imperial College London in 2006 In 2006, he joined as a Lecturer with the School of Civil and Building Engineering, Loughborough University, U.K., and was promoted to a Senior Lecturer in 2010 and a Professor of intelligent transportation systems in 2013. He has authored over 100 technical papers in international refereed journals and conference proceedings. His current research interests include high-accuracy and integrity land vehicle navigation, autonomous navigation, and sensor fusion. Prof. Quddus is a member of the U.S. Transportation Research Board, the British Computer Society, the EPSRC, and the Universities Transport Study Group, U.K.

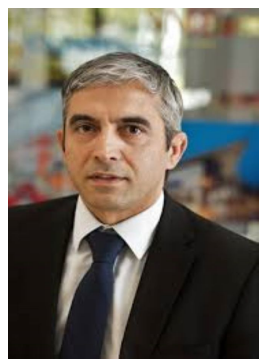

Mehrdad Dianati was a Professor with the 5G Innovation Centre (5GIC), University of Surrey, where he is currently a Visiting Professor. He is also a Professor of autonomous and connected vehicles with the Warwick Manufacturing Group (WMG), University of Warwick. He has been involved in a number of national and international projects as the project leader and work-package leader in recent years. $\mathrm{He}$ has worked in the industry for more than 9 years as a senior software/hardware developer and the director of R\&D. He frequently provides voluntary services to the research community in various editorial roles; for example, he has served as an Associate Editor for the IEEE TRANSACTIONS ON VEHICULAR TECHNOLOGY, IET Communications, and Wileys Journal of Wireless Communications and Mobile.

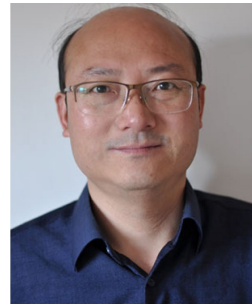

Wen-Hua Chen (M'00-SM'06-F'18) currently holds Professor in Autonomous Vehicles in the Department of Aeronautical and Automotive Engineering at Loughborough University, UK. He received his M.Sc. and Ph.D. degrees from the Department of Automatic Control at Northeast University, China, in 1989 and 1991, respectively. From 1991 to 1996, he was a Lecturer in the Department of Automatic Control at Nanjing University of Aeronautics and Astronautics, China. He held a research position and then a Lectureship in control engineering in the Centre for Systems and Control at University of Glasgow, UK, from 1997 to 2000. He has published three books and 250 papers in journals and conferences. His research interests include the development of advanced control strategies and their applications in aerospace engineering, particularly in unmanned aircraft systems. He is a Fellow of the Institution of Engineering and Technology, the Institution of Mechanical Engineers, and IEEE. 